\title{
Downregulation of miR-193a-3p via targeting cyclin D1 in thyroid cancer
}

\author{
XIAO-JIAO LI ${ }^{1 *}$, RONG WEN ${ }^{2 *}$, DONG-YUE WEN ${ }^{2}$, PENG LIN ${ }^{2}$, DENG-HUA PAN ${ }^{2}$, LI-JIE ZHANG ${ }^{2}$, \\ YU HE ${ }^{2}$, LIN SHI ${ }^{3}$, YONG-YING QIN ${ }^{2}$, YUN-HUI LAI ${ }^{3}$, JING-NI LAI $^{2}$, JUN-LIN YANG $^{2}$, \\ QIN-QIAO LAI ${ }^{3}$, JUN WANG $^{3}, \mathrm{JUN} \mathrm{MA}^{3}, \mathrm{HONG} \mathrm{YANG}^{2}$ and YU-YAN PANG ${ }^{4}$ \\ ${ }^{1}$ Department of Positron Emission Tomography-Computed Tomography (PET-CT) and \\ ${ }^{2}$ Ultrasonics Division of Radiology Department, First Affiliated Hospital of Guangxi Medical University, \\ Nanning, Guangxi Zhuang Autonomous Region 530021; ${ }^{3}$ Department of Pathology, \\ Second Affiliated Hospital of Guangxi Medical University, Nanning, \\ Guangxi Zhuang Autonomous Region 530007; ${ }^{4}$ Department of Pathology, \\ First Affiliated Hospital of Guangxi Medical University, Nanning, \\ Guangxi Zhuang Autonomous Region 530021, P.R. China
}

Received May 4, 2019; Accepted April 9, 2020

DOI: $10.3892 / \mathrm{mmr} .2020 .11310$

\begin{abstract}
Thyroid cancer (TC) is a frequently occurring malignant tumor with a rising steadily incidence. microRNA (miRNA/miR)-193a-3p is an miRNA that is associated with tumors, playing a crucial role in the genesis and progression of various cancers. However, the expression levels of miR-193a-3p and its molecular mechanisms in TC remain to be elucidated. The present study aimed to probe the expression of miR-193a-3p and its clinical significance in TC, including its underlying molecular mechanisms. Microarray and RNA sequencing data gathered from three major databases, specifically Gene Expression Omnibus (GEO), ArrayExpress and The Cancer Genome Atlas (TCGA) databases, and the relevant data from the literature were used to examine miR-193a-3p expression. Meta-analysis was also conducted to evaluate the association between clinicopathological parameters and miR-193a-3p in 510 TC and 59 normal samples from the TCGA database. miRWalk 3.0, and the TCGA and GEO databases
\end{abstract}

Correspondence to: Professor Hong Yang, Ultrasonics Division of Radiology Department, First Affiliated Hospital of Guangxi Medical University, 6 Shuangyong Road, Nanning, Guangxi Zhuang Autonomous Region 530021, P.R. China

E-mail: yanghong@gxmu.edu.cn

Professor Yu-Yan Pang, Department of Pathology, First Affiliated Hospital of Guangxi Medical University, 6 Shuangyong Road, Nanning, Guangxi Zhuang Autonomous Region 530021, P.R. China E-mail: hornor159@126.com

*Contributed equally

Key words: thyroid cancer, microRNA-193a-3p, cyclin D1, in-house dual luciferase assay, meta-analysis were used to predict the candidate target genes of miR-193a-3p. Gene Ontology, Kyoto Encyclopedia of Genes and Genomes and protein-protein interaction network enrichment analyses were conducted by using the predicted candidate target genes to investigate the underlying carcinogenic mechanisms. A dual luciferase assay was performed to validate the targeting regulatory association between the most important hub gene cyclin D1 (CCND1) and miR-193a-3p. miR-193a-3p expression was considerably downregulated in TC compared with in the non-cancer controls $(\mathrm{P}<0.001)$. The area under the curve of the summary receiver operating characteristic was 0.80 . Downregulation of miR-193a-3p was also significantly associated with age, sex and metastasis $(\mathrm{P}=0.020,0.044$ and 0.048 , respectively). Bioinformatics analysis indicated that a low miR-193a-3p expression may augment CCND1 expression to affect the biological processes of TC. In addition, CCND1, as a straightforward target, was validated through a dual luciferase assay. miR-193a-3p and CCND1 may serve as prognostic biomarkers of TC. Finally, miR-193a-3p may possess a crucial role in the genesis and progression of TC by altering the CCND1 expression.

\section{Introduction}

Thyroid cancer (TC) is a frequently occurring malignant tumor with steadily increasing incidence (1-5). In 2018, the American Cancer Society predicted that there would be 53,990 newly diagnosed patients with TC that year (6). Based on histopathological examination, TC is divided into 4 subtypes: Anaplastic thyroid carcinoma, papillary thyroid carcinoma, medullary thyroid cancer and follicular thyroid cancer (7-9). Different hypotypes of TC lead to various clinical outcomes. A number of studies have reported that intricate biological processes participating in the interactions between polygenes may contribute to the occurrence and progression of TC (10-12). Previous studies have suggested that hsa-miR-200a-5p and 
hsa-miR-181a-2-3p could be applied for latent diagnostic and prognostic biomarkers of TC $(13,14)$. Nevertheless, the clinical characteristics of molecular profiles in TC have yet to be elucidated. Therefore, to further understand the molecular mechanisms of TC, an multi-method study may offer novel insight for TC prevention and treatment (15).

MicroRNAs (miRNAs/miRs) contain $~ 19-25$ nucleotides and are endogenous non-coding RNAs with gene-regulating functions (16-19). miRNAs are critical in the biological processes of diversified human carcinomas. For instance, they participate in extensive biological actions, including cell proliferation, invasion and apoptosis $(18,20-23)$. Previous studies have demonstrated that miR-193a-3p is a neoplasm suppressor in various carcinomas, including hepatocellular cancer (24), gastric cancer $(25,26)$, lung carcinoma (27), breast carcinoma (28) and colorectal carcinoma $(29,30)$. To the best of the authors' knowledge, thus far only one study has investigated the association between miR-193a-3p and medullary thyroid carcinoma (31); however, a limitation of this study was the small sample size, which prevented a comprehensive description and analysis of TC. To further comprehend the potential molecular mechanisms, it is necessary to examine miR-193a-3p expression in TC using a larger volume of data.

Cyclin D1 (CCND1), a member of the highly conserved cyclin family, can function as a modulator of cyclin-dependent kinase (CDK) 4 or CDK6, and has an important role in adjusting the progression of the cell cycle $(32,33)$. Previous studies revealed that CCND1 has a crucial role in promoting proliferation, migration and tumor metastasis $(34,35)$ in cancers such as lung cancer (33), gastric cancer (36) and renal cell cancer (37). CCND1 has been reported to exhibit a high expression in TC tissues and to be associated with invasive behavior (38).

The present study examined TC specimen information from The Cancer Genome Atlas (TCGA) to assess the expression levels of miR-193a-3p and CCND1, and their association with clinical parameters. The data of miR-193a-3p and CCND1 were extracted and validated via ArrayExpress databases and the Gene Expression Omnibus (GEO). Then, the candidate target genes of miR-193a-3p were identified using miRWalk 3.0 and overlapped with the upregulated genes in TC identified using TCGA database. The miR-193a-3p candidate target genes were further investigated using Gene Ontology (GO), Kyoto Encyclopedia of Genes and Genomes (KEGG) pathway analysis and protein-protein interaction (PPI) network enrichment analyses. According to the bioinformatics analysis of the present study, a hub gene was found in TC, and the targeting regulatory relations between the hub gene and miR-193a-3p were confirmed by a dual luciferase assay. By performing a meta-analysis and bioinformatics analysis, the present study revealed the latent molecular mechanisms of miR-193a-3p, as well as its associations with the identified hub gene, which may lead to TC.

\section{Materials and methods}

miR-193a-3p expression in TC from public databases and the literature. TCGA is a pool of molecular datasets for at least 30 types of cancers, including TC (39). The present study explored TCGA to determine miR-193a-3p expression in TC tissues. miRNA expression data of $510 \mathrm{TC}$ and 59 normal samples were acquired, along with the accompanying clinical information, from TCGA, up to December 1, 2018 (40). Chip datasets (GSE40807, GSE62054 and GSE73182) from GEO were also searched to examine the profiling of miR-193a-3p expression in TC (41-43). The following keywords were used: (papillary OR medullary OR follicular OR anaplastic OR thyroid) AND (carcinoma OR cancer OR adenocarcinoma OR tumor OR neoplas* OR malignan*) AND (microRNA OR miR OR miRNA), where "*' refers to a wildcard search. The public database of microarray gene expression, ArrayExpress (E-MTAB-736) (44) was also used. In addition, literature that included miR-193a-3p expression in TC was searched for in 13 online databases: Wiley Online Library (https://onlinelibrary.wiley.com), Ovid (https://www.ovid.com), LILACS (https://lilacs.bvsalud.org), Web of Science (http://www. isiknowledge.com), PubMed (https://www.ncbi.nlm.nih. gov/pubmed), Science Direct (https://www.sciencedirect.com), Cochrane Central Register of Controlled Trials (https://www. cochranelibrary.com), EMBASE (https://www.embase.com), Google Scholar (https://scholar.google.com), Chong Qing VIP (http://lib.cqvip.com), Wan Fang (http://www.wanfangdata. com), Chinese CNKI (https://www.cnki.net) and the China Biology Medicine Disc (http://www.sinomed.ac.cn). The studies needed to meet the following requirements for inclusion: i) The miR-193a-3p expression data in TC could be tested in humans; and ii) the relevant information of miR-193a-3p could be abstracted from the literature. All expression data and clinical information connected to miR-193a-3p were extracted.

Comprehensive statistical analysis of TCGA, GEO, ArrayExpress and the literature. The expression levels of both TC and non-cancerous samples were visualized using scatter diagrams and receiver operating characteristic (ROC) curves created using GraphPad Prism 6.0 (GraphPad Software, Inc.). Student's t-test was performed to compare the expression of $\mathrm{TC}$ and non-cancer samples, as well as the association between clinicopathological parameters and miR-193a-3p from TCGA database. One-way analysis of variance was used for three or more groups. All miR-193a-3p expression data abstracted from various databases were normalized to $\log 2$, and $\mathrm{P}<0.05$ was considered to indicate a statistically significant difference. An all-sided analysis was performed by employing STATA 12.0 software (StataCorp LLC). The pooled standard mean difference (SMD) was applied to evaluate miR-193a-3p expression. $\chi^{2}$ and $I^{2}$ tests were computed to assess the heterogeneity within the meta-analysis. The Mantel-Haenszel fixed-effects model was selected if there was no prominent heterogeneity $\left(\mathrm{I}^{2}<50 \%\right)$. Otherwise, a random-effects model was applied $\left(\mathrm{I}^{2}>50 \%\right)(45)$; meanwhile, heterogeneity analysis was performed to discover the sources of heterogeneity. Subsequently, a summary (s)ROC was created to indicate the ability of miR-193a-3p to identify TC compared with a normal sample (46-48).

Prediction of miR-193a-3p candidate target genes and their molecular functions. To further search for the latent molecular functions of miR-193a-3p in TC, bioinformatics analysis was performed. First, the target genes of miR-193a-3p were predicted using 12 platforms [miRWalk 2.0 (version 2; http://zmf.umm.uni-heidelberg.de/apps/zmf/mirwalk2), PITA (version 6; https://genie.weizmann.ac.il/pubs/mir07/mir07_exe. 
html), PicTar2 (https://pictar.mdc-berlin.de), RNAhybrid 2.1 (https://bibiserv.cebitec.uni-bielefeld.de/rnahybrid), MicroT4 v4.0 (version 5; http://diana.imis.athena-innovation.gr), miRDB 4.0 (http://www.mirdb.org), miRanda-rel2010 (http://www. microrna.org/microrna/getDownloads.do), TargetScan 7.2 (version 7.2; http://www.targetscan.org), RNA22 v2 (https://cm. jefferson.edu/rna22-full-sets-of-predictions), miRBridge (http://www.ncbi.nlm.nih.gov/pubmed/?term=20385095), miRNAMap (ftp://mirnamap.mbc.nctu.edu.tw/miRNAMap2) and miRMap (https://mirmap.ezlab.org)]. The predicted genes exhibited in the 12 platforms were selected: 3,984 candidate target genes. Log2 conversion and normalization was performed for the gene chips and RNA sequencing from the GEO and TCGA databases. Differentially expressed genes in microarrays and RNA sequencing data were also screened out by the limma package (version 3.42.2) of $\mathrm{R}$ language (R version 3.6.2; https://www.r-project.org) (49). Integration of differentially expressed genes identified from gene chips and RNA sequencing was conducted via RobustRankAggreg (version 1.1; https://cran.r-project.org/web/packages/Robust RankAggreg). The overexpressed genes in TC from the TCGA and GEO databases were calculated by selecting the following criteria: $\mathrm{P}$-adj<0.05 and any upregulated genes with a $\log 2$-fold change $(\mathrm{FC})>1$. The candidate target genes obtained with the online prediction tools in TCGA and GEO were then overlapped and presented as Venn diagrams (50). Then, the overlapping target genes were used to study molecular mechanisms via GO (51), KEGG pathway (52), Disease Ontology (DO) (53) and PPI network analyses. Bioinformatics analyses were implemented using clusterprofiler package (version 3.10) of $\mathrm{R}$ language (54). The ggplot2 package (version 3.3.0) from $\mathrm{R}$ language was utilized to visualize the results of the functional analysis (55). STRING 11.0 (http://string.embl.de) was also used to generate a PPI network, which revealed the connection among the overlapping related genes (56).

Expression of CCND1 in TC samples from the TCGA and GEO databases. The clinical value of CCND1, whose expression data was extracted from GEO and TCGA, was further explored. CCND1 expression data and its clinical information were acquired from TCGA. The GEO database was also mined to obtain chip datasets from TC samples using the following keywords: (papillary OR medullary OR follicular OR anaplastic OR thyroid) AND (carcinoma OR tumor OR cancer OR adenocarcinoma OR neoplas* OR malignan*) AND (Cyclin D1 OR G1/S-Specific Cyclin-D1 OR PRAD1 Protein OR B-Cell CLL/Lymphoma 1 OR U21B31 OR BCL-1 Oncogene OR BCL1 OR PRAD1 Oncogene OR Parathyroid Adenomatosis 1 OR D11S287E OR B-Cell Lymphoma 1 Protein). Filtering condition: Series [Entry type], Homo sapiens [Organism]. A total of 13 datasets were obtained: GSE3678, GSE6004, GSE6339, GSE9115, GSE27155, GSE29265, GSE33630, GSE35570, GSE50901, GSE53072, GSE53157, GSE58689 and GSE65144 (57-67). Statistical analysis of CCND1 was also performed. The methods used were the same as those used above for evaluating the expression of miR-193a-3p.

Cell culture and transfection. The 293 cell line was obtained from Huzhou Hippo Biotechnology Co., Ltd. and cultured in
DMEM (Gibco; Thermo Fisher Scientific, Inc.) supplemented with $10 \%$ fetal bovine serum (Gibco; Thermo Fisher Scientific, Inc.) and $1 \%$ penicillin/streptomycin. Cells were cultured in an incubator at $37^{\circ} \mathrm{C}$ with $5 \% \mathrm{CO}_{2}$. miR-193a-3p mimic (5'-AAC UGGCCUACAAAGUCCCAGU-3'), miR-146b-5p mimic (5'-UGAGAACUGAAUUCCAUAGGCUG-3') and mimic control (5'-UUUGUACUACACAAAAGUACUG-3') were synthesized and obtained from Hippobio Co., Ltd. miR-193a-3p mimic, miR-146b-5p mimic and their mimic control (all $75 \mathrm{pM})$ were transfected into $2.5 \times 10^{5} 293$ cells/well using Lipofectamine ${ }^{\circledR} 3000$ reagent (Invitrogen; Thermo Fisher Scientific, Inc.), according to the manufacturer's protocol. Transfection efficiency was measured by reverse transcription-quantitative PCR (RT-qPCR) after $48 \mathrm{~h}$.

RNA extraction and $R T-q P C R$. Cellular RNA was extracted from 293 cells with TRIzol ${ }^{\odot}$ reagent (Tiangen Biotech Co.,Ltd.). RevertAid Reverse Transcriptase (Invitrogen; Thermo Fisher Scientific, Inc.) was used to reverse transcribe miRNA according to the manufacturer's protocol. The expression of miR-193a-3p and miR-146b-5p was performed by qPCR using PerfectStart Green qPCR SuperMix (Transgen Biotech Co., Ltd.) on an ABI Q1 RT-qPCR System (Applied Biosystems; Thermo Fisher Scientific, Inc.). U6 was used as an internal reference. The relevant primer sequences were as follows: miR-193a-3p forward, 5'-GCCGAGAACTGGCCTACAAA-3' and reverse, 5'-CTCAACTGGTGTCGTGGAGTCGGCA ATTCAGTTGAGACTGGGAC-3'; miR-146b-5p forward, 5'-GCCGAGTGAGAACTGAATTCC-3' and reverse, 5'-CTC AACTGGTGTCGTGGAGTCGGCAATTCAGTTGAGCAG CCTAT-3'; U6 forward, 5'-CTCGCTTCG GCAGCACA-3' and reverse, 5'-AACGCTTCA CGAATTTGCGT-3'. miR-193a-3p and $\mathrm{miR}-146 \mathrm{~b}-5 \mathrm{p}$ expression levels were calculated by the $2^{-\Delta \Delta \mathrm{Cq}}$ method (68).

Validation of the association between miR-193a-3p and the hub gene. The protein with the most connections was selected for further investigation. 293 cells exhibit a high transfection efficiency and were thus selected for the luciferase activity assay. The wild-type (Wt) CCND1-3' untranslated region (UTR) and seed region mutant (Mut) of the miR-193a-3p binding site were subcloned into the pGL3 system (Promega Corporation). Its corresponding positive control [Wt tumor necrosis factor receptor-associated factor 6 (TRAF6)-3' UTR + miR-146b-5p] was also subcloned into the pGL3 system (69,70). Subsequently, $1 \times 10^{5} 293$ cells/well were co-transfected with $100 \mathrm{ng}$ Wt CCND1-3' UTR or Wt TRAF6-3' UTR vector and $400 \mathrm{ng}$ miRNA mimic or negative control (NC) plasmid using X-tremeGENE ${ }^{\text {TM }}$ HP DNA transfection reagent [Roche Diagnostics (Shanghai) Co., Ltd.]. Then, 2 days following incubation, firefly luciferase activity was assessed using a Dual-Luciferase Reporter assay system (Promega Corporation) in accordance with the manufacturer's protocols. Firefly luciferase activity was normalized to Renilla luciferase activity. Student's t-test was used to compare mimics and mimic control for each luciferase plasmid. Pearson's correlation analysis was also conducted to analyze the relationship between miR-193a-3p and CCND1, and $\mathrm{P}<0.05$ was considered to indicate a statistically significant difference. Hub gene expression between the TC samples and the normal 

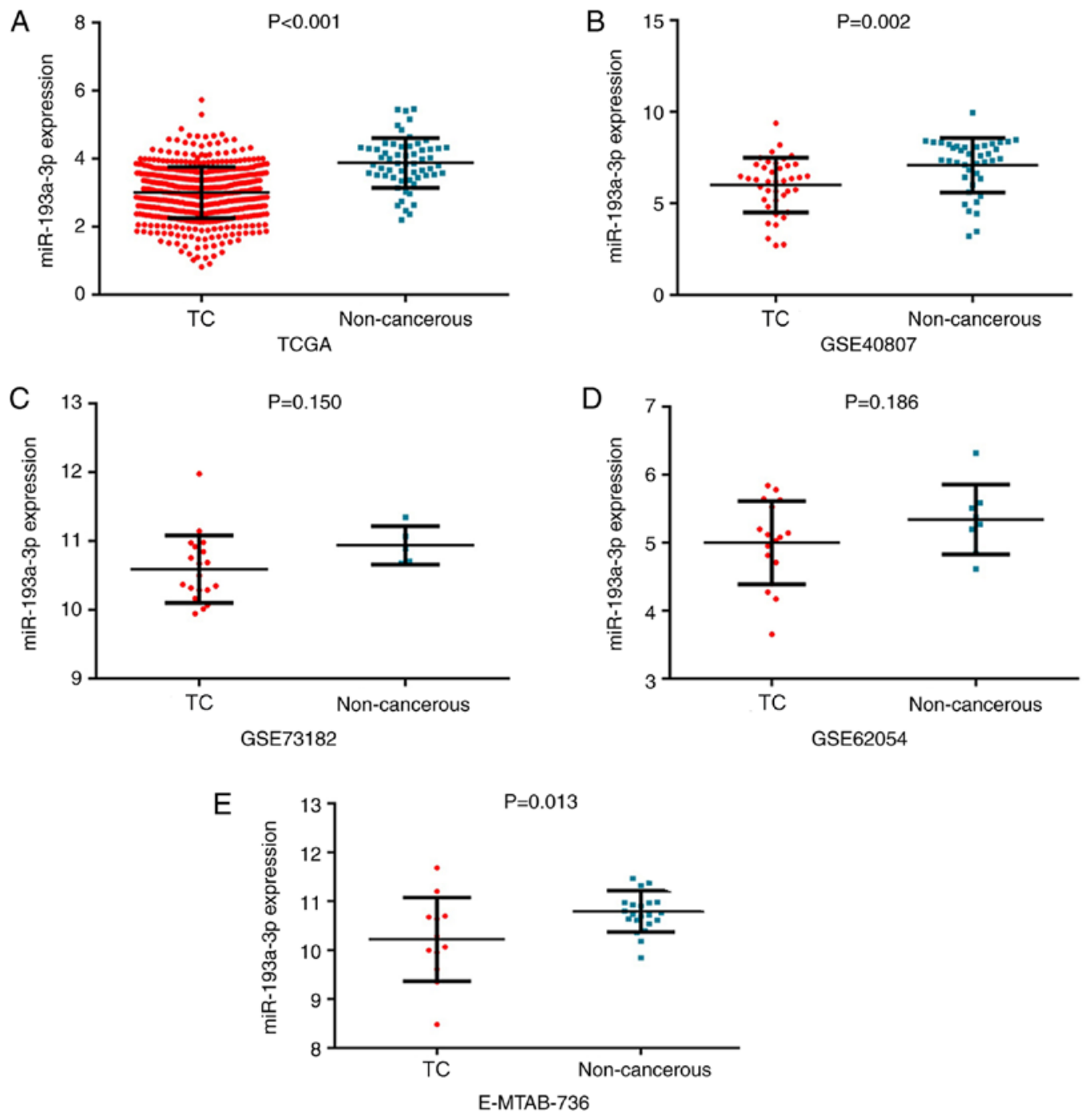

Figure 1. Validation of miR-193a-3p expression in different databases. (A) TCGA, (B) GSE40807, (C) GSE73182, (D) GSE62054 and (E) E-MTAB-736. miR, microRNA; TC, thyroid cancer; TCGA, The Cancer Genome Atlas.

samples was explored via immunohistochemistry using The Human Protein Atlas (71).

\section{Results}

Downregulated miR-193a-3p expression in TC and its clinical significance from TCGA. The expression of miR-193a-3p in the TC group was $3.00 \pm 0.75$ and that in the non-cancerous group was $3.88 \pm 0.73$. miR-193a-3p exhibited significantly reduced expression in $510 \mathrm{TC}$ samples compared with in 59 non-cancer controls ( $\mathrm{P}<0.001$; Fig. 1A). The ROC curve of miR-193a-3p was computed with an area under the curve (AUC) of 0.802 $(\mathrm{P}<0.001$; Fig. 2A), which reflected the moderate value of miR-193a-3p to differentiate TC from the non-cancerous groups. The cut-off value was 3.444 (sensitivity $71.2 \%$ and specificity $79.7 \%$ ). miR-193a-3p expression and clinical parameters are displayed in Table I. The miR-193a-3p levels in females were notably greater than those in males $(\mathrm{P}=0.004)$. miR-193a-3p was markedly decreased in individuals $\leq 60$ years of age compared with those $>60$ years $(\mathrm{P}=0.020)$. In addition, the number of distant metastases showed a negative association with miR-193a-3p expression levels $(\mathrm{P}=0.048)$.
miR-193a-3p expression in TC verified via GEO, ArrayExpress and literature analyses. Subsequently, in other online databases, data from 4 chips (GSE40807, GSE62054, GSE73182 and E-MTAB-736) were acquired from the GEO and ArrayExpress databases, which provided the expression information on miR-193a-3p in 88 TC and 75 non-cancerous tissues (Table II). The literature was also searched in 13 online databases, but the data from one study could not be extracted. All 4 datasets obtained from the GEO and ArrayExpress databases revealed that miR-193a-3p expression levels in TC were greater compared with non-TC controls, although two of these four datasets had P-values $>0.05$ (Fig. 1B-E). ROC analysis was also used to estimate the capacity of miR-193a-3p in distinguishing TC from non-cancer tissues (Fig. 2B-E); three of the four chips demonstrated that miR-193a-3p had a moderate diagnostic value for TC. miR-193a-3p expression data from TCGA, GEO and ArrayExpress, which provided $598 \mathrm{TC}$ and 134 non-cancerous samples, were combined for meta-analysis. The SMD of miR-193a-3p was -1.00 [P<0.001; 95\% CI, (-1.21, -0.79); Fig. 3A] via the fixed-effects model, and the heterogeneity test was $\mathrm{P}=0.46\left(\mathrm{I}^{2}=0 \%\right)$. The AUC of the sROC was 0.80 (95\% CI, 0.76-0.83), indicating 

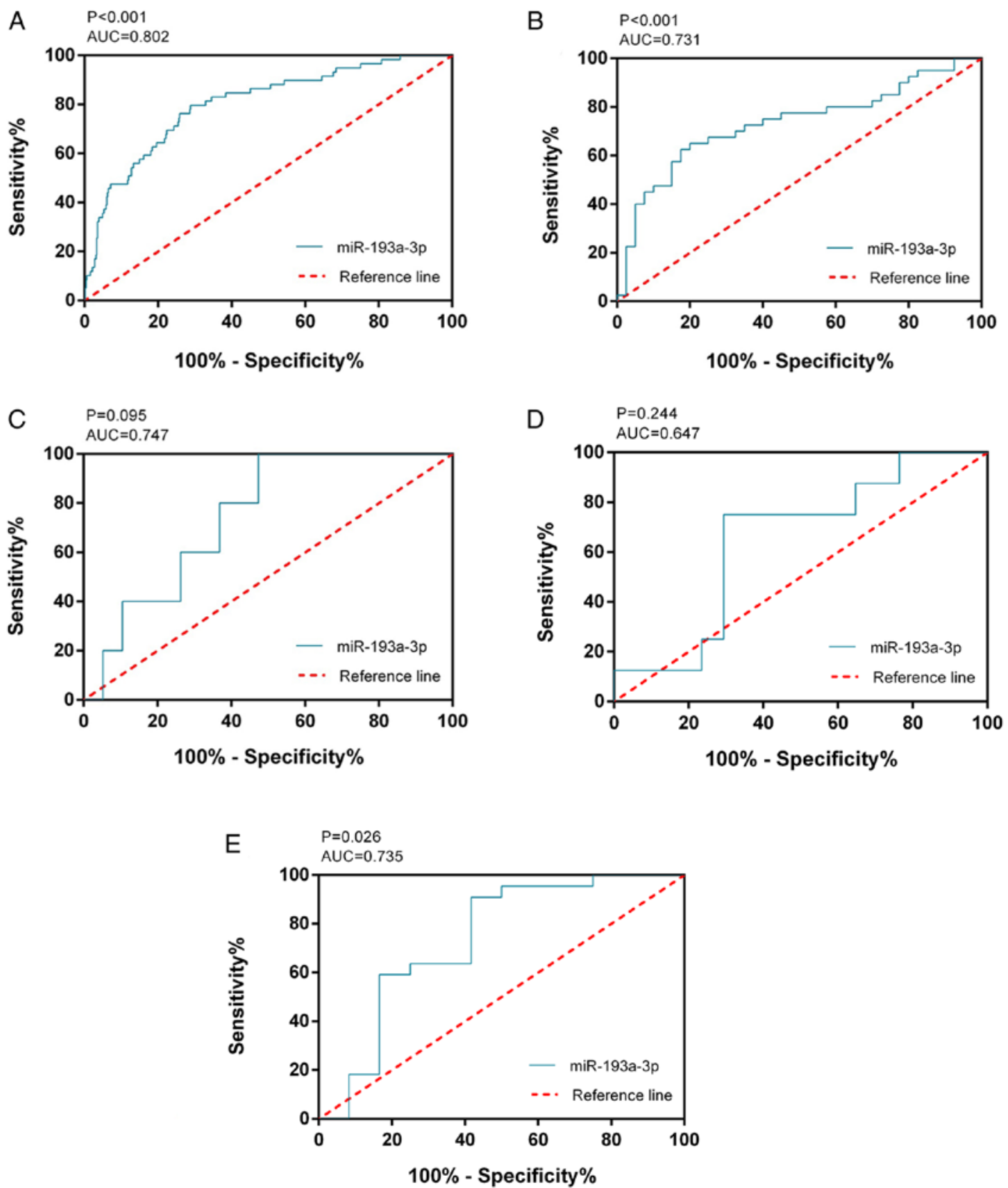

Figure 2. Receiver operating characteristic curve analysis of miR-193a-3p from TCGA and GEO databases. (A) TCGA, (B) GSE40807, (C) GSE73182, (D) GSE62054 and (E) E-MTAB-736 . miR, microRNA; TCGA, The Cancer Genome Atlas; GEO, Gene Expression Omnibus; AUC, area under curve.

a moderate ability to differentiate TC from non-cancer samples. The combined sensitivity and specificity were 0.69 (95\% CI, 0.59-0.78) and 0.82 (95\% CI, 0.67-0.91), respectively (Fig. 3B). Publication bias was performed and showed that there was no conspicuous publication bias among the datasets (Begg's test and Egger's test were 0.81 and 0.16, respectively; Fig. 3C and D).

GOannotation, KEGG pathway,DO andPPInetwork analysis. The prediction of the candidate target genes of miR-193a-3p was conducted using miRWalk 3.0, in which 3,984 potential mRNA candidate target genes were discovered. Next, 1,490 and 132 genes with elevated expression were determined from the TCGA and GEO databases, respectively. A total of 28 overlapping genes were eventually identified (Fig. 4A). The properties of these target genes were analyzed using the clusterprofiler $\mathrm{R}$ package. The results indicated that 'response to nutrient levels' was the most significantly enriched biological process (BP); with respect to cellular components (CC), the genes were mainly enriched in the 'lamellar body'. In terms of molecular function (MF), 'sulfur compound binding' was the most enriched (Fig. 5A-C; Table III). However, no KEGG pathways were significantly enriched with these genes. DO analysis was also performed to discover related diseases caused by these genes. The results identified that "cell type benign neoplasm' was the most enriched (Fig. 5D; Table III). Then, the target genes were put into the STRING online website to construct a protein-protein interaction network (Fig. 4B). In the present study, CCND1 attracted attention as a hub gene, as it had the most connections among the proteins; therefore, the 
Table I. Association between miR-193a-3p expression and clinicopathological variables in TC from The Cancer Genome Atlas.

\begin{tabular}{|c|c|c|c|c|}
\hline \multirow[b]{2}{*}{ Clinicopathological feature } & \multirow[b]{2}{*}{ Cases } & \multicolumn{3}{|c|}{ miR-193a-3p expression } \\
\hline & & Mean \pm SD & $\mathrm{t} / \mathrm{F}$ value & P-value \\
\hline \multicolumn{5}{|l|}{ Tissue } \\
\hline Non-cancerous tissues & 59 & $3.88 \pm 0.73$ & 8.468 & $<0.001$ \\
\hline $\mathrm{TC}$ & 510 & $3.00 \pm 0.75$ & & \\
\hline \multicolumn{5}{|l|}{ Sex } \\
\hline Male & 134 & $2.89 \pm 0.73$ & -2.022 & 0.044 \\
\hline Female & 368 & $3.04 \pm 0.76$ & & \\
\hline \multicolumn{5}{|l|}{ Pathologic tumor grade } \\
\hline Stage I-II & 334 & $2.99 \pm 0.78$ & -0.459 & 0.647 \\
\hline Stage III-IV & 166 & $3.02 \pm 0.72$ & & \\
\hline \multicolumn{5}{|l|}{ Age } \\
\hline$<60$ & 383 & $3.04 \pm 0.75$ & 2.336 & 0.020 \\
\hline$\geq 60$ & 119 & $2.86 \pm 0.77$ & & \\
\hline \multicolumn{5}{|l|}{ TNM stage } \\
\hline $\mathrm{T} 1-\mathrm{T} 2$ & 310 & $3.01 \pm 0.77$ & 0.433 & 0.665 \\
\hline T3-T4 & 190 & $2.98 \pm 0.74$ & & \\
\hline \multicolumn{5}{|l|}{ Pathological lymph node } \\
\hline No & 229 & $2.96 \pm 0.75$ & -1.074 & 0.283 \\
\hline Yes & 223 & $3.04 \pm 0.73$ & & \\
\hline \multicolumn{5}{|l|}{ Metastasis } \\
\hline No & 278 & $3.03 \pm 0.76$ & 1.990 & 0.048 \\
\hline Yes & 9 & $2.52 \pm 0.95$ & & \\
\hline \multicolumn{5}{|l|}{ Ethnicity } \\
\hline Caucasian & 330 & $3.00 \pm 0.77$ & 2.236 & 0.108 \\
\hline African-American & 27 & $2.69 \pm 0.73$ & & \\
\hline Asian & 52 & $3.01 \pm 0.68$ & & \\
\hline
\end{tabular}

miR, microRNA; TC, thyroid cancer.

Table II. General characteristics of included microarray and RNA sequencing datasets.

\begin{tabular}{|c|c|c|c|c|c|}
\hline Authors, year & Country & Dataset & Cancer group & Non-cancer groups & Refs. \\
\hline TCGA, 2018 & USA & TCGA & 510 & 59 & - \\
\hline Lassalle et al, 2016 & France & GSE40807 & 40 & 40 & $(42)$ \\
\hline Stokowy et al, 2014 & Norway & GSE62054 & 17 & 5 & Unpublished data \\
\hline Minna et al, 2016 & Italy & GSE73182 & 19 & 5 & $(43)$ \\
\hline Rossing et al, 2012 & Denmark & E-MTAB-736 & 12 & 22 & (44) \\
\hline
\end{tabular}

TCGA, The Cancer Genome Atlas.

targeting association between CCND1 and miR-193a-3p was selected for further investigation.

Overexpression of CCND1 in TC and its clinical significance. The expression value and clinical significance of CCND1 in TC was verified using data from two different sources (TCGA and GEO databases; Table IV). The expression of CCND1 in the TC group from TCGA database was $13.36 \pm 0.74$ and that in the non-cancerous group was $11.66 \pm 0.49$. CCND1 was significantly overexpressed in $513 \mathrm{TC}$ samples compared with 59 non-cancerous samples $(\mathrm{P}<0.001$; Fig. 6A). The AUC for upregulated CCND1 expression in TC was 0.965 $(\mathrm{P}<0.001$; Fig. 7A), which indicated the high capacity of the expression value of CCND1 to differentiate TC from normal 


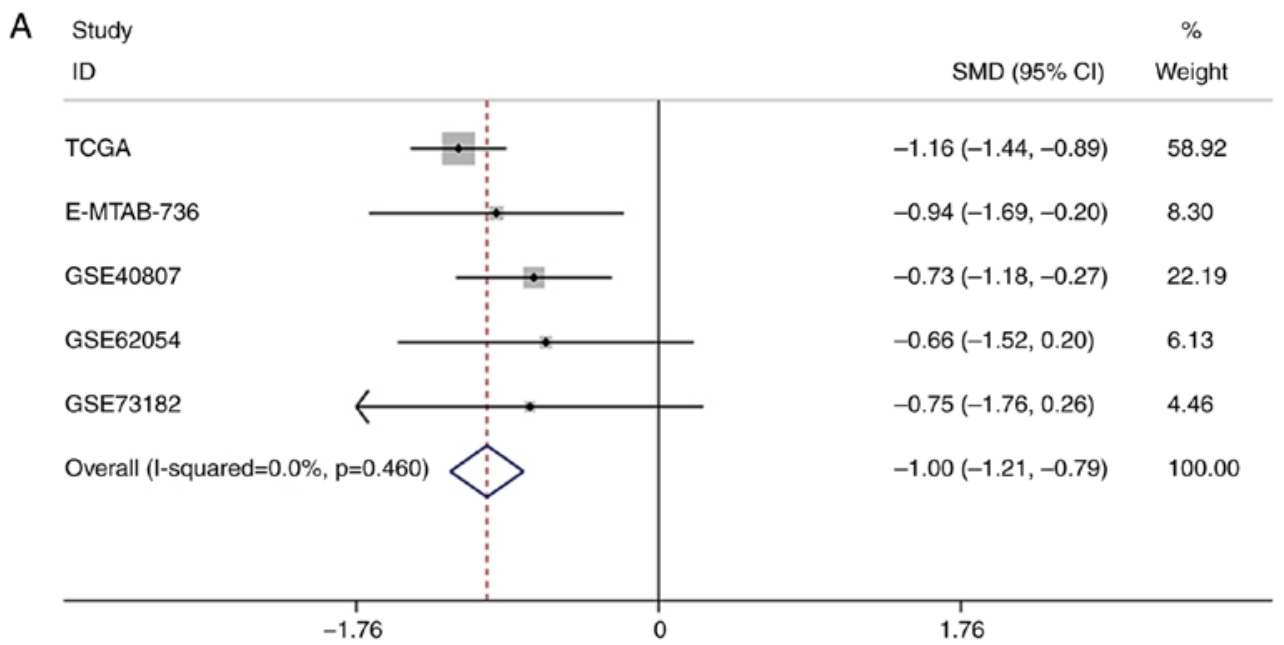

B
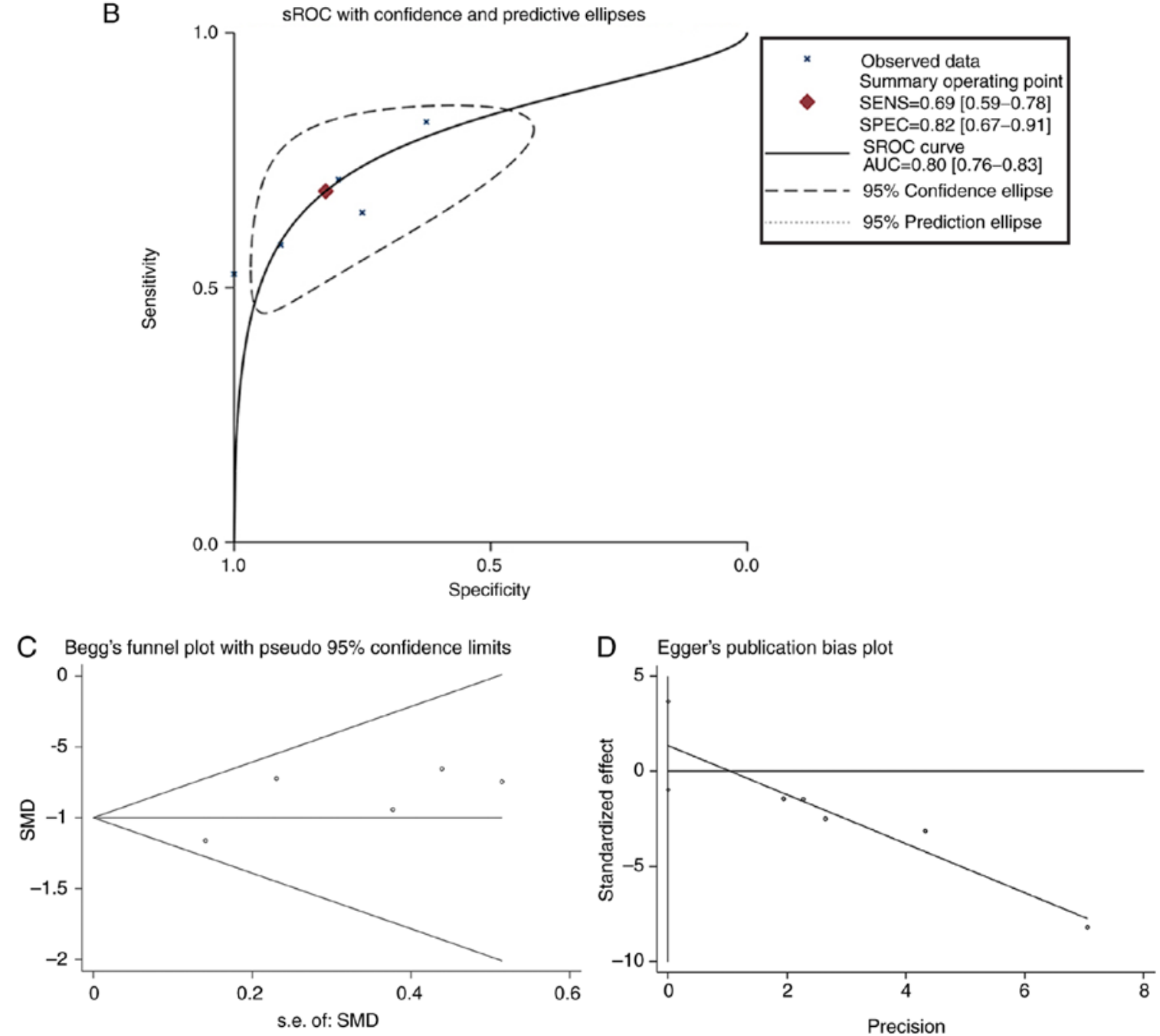

Figure 3. Meta-analysis of miR-193a-3p in TC. (A) Forest plot curve for evaluating miR-193a-3p expression in TC compared with non-cancerous tissues, the pooled SMD was -1.00 [P<0.001; 95\% CI, -1.21-(-0.79)]. (B) AUC of sROC Curve was 0.80 (95\% CI, 0.76-0.83), which indicated a moderate ability to differentiate TC from non-cancer samples. The pooled sensitivity and specificity were 0.69 (95\% CI, 0.59-0.78) and 0.82 (95\% CI, 0.67-0.91), respectively. (C) Begg's test. (D) Egger's test. miR, microRNA; TC, thyroid cancer; SMD, standard mean difference; CI, confidence interval; AUC, area under curve; sROC, summary receiver operating characteristic; TCGA, The Cancer Genome Atlas.

samples. The cut-off value was 12.628 (sensitivity $86.9 \%$ and specificity 100\%). Differential CCND1 expression was not detected for the clinical pathological parameters analyzed. The associations between the expression of CCND1 and clinical pathological parameters are summarized in Table V. The expression value of CCND1 was also calculated from the chip arrays of the GEO database, which included $384 \mathrm{TC}$ and 316 non-cancer controls (GSE3678, GSE6004, 

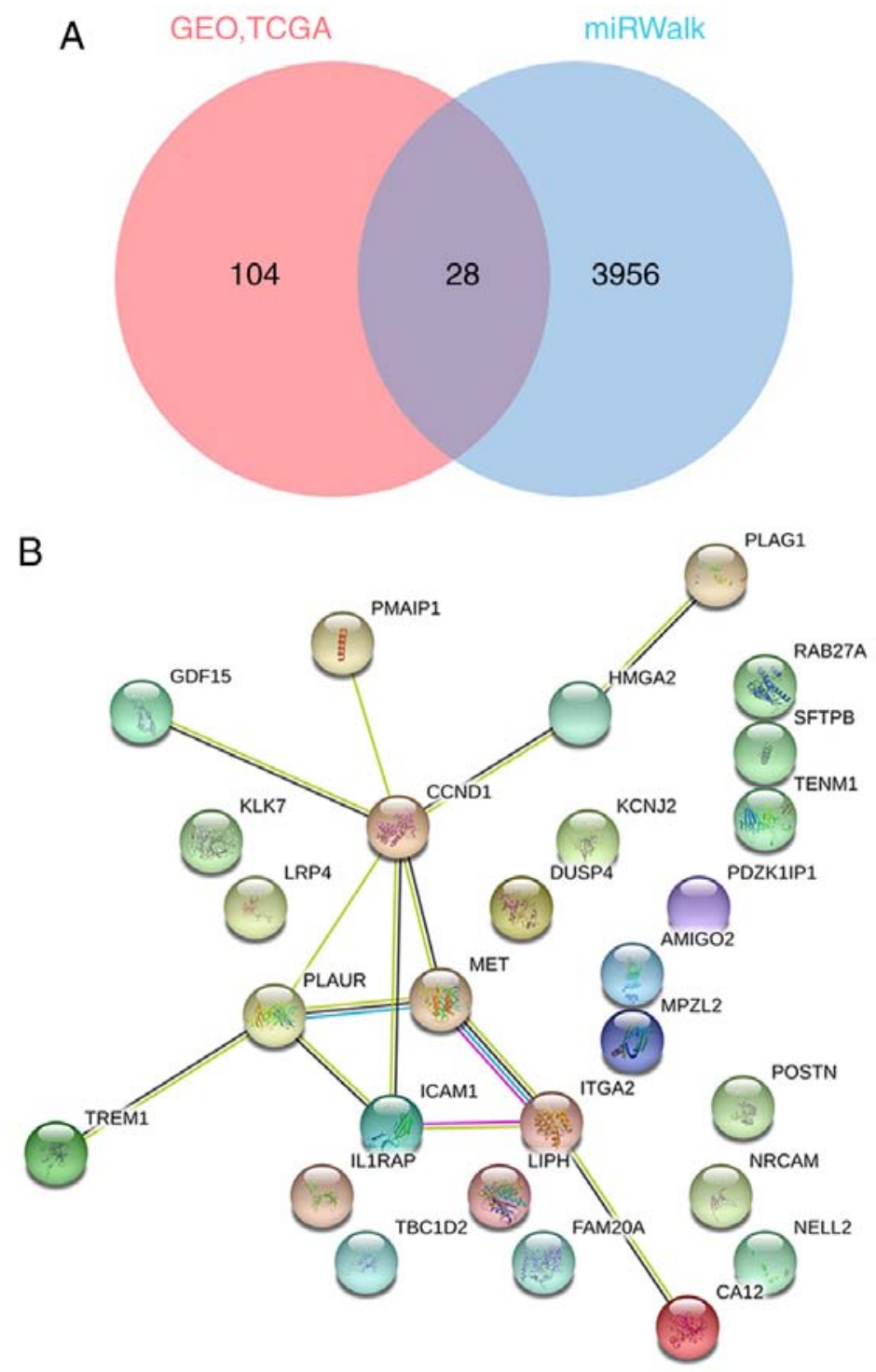

Figure 4. Identification of miR-193a-3p -related target genes. (A) Venn diagram for overlap among GEO, TCGA and miRWalk. (B) Protein-protein interaction network of the target genes. Each node represents a protein. The lines represent the interaction between the proteins. Different line colors correspond to different types of interaction (blue, from curated databases; pink, experimentally determined; yellow, textmining; black, co-expression). GEO, Gene Expression Omnibus; TCGA, The Cancer Genome Atlas.

GSE6339, GSE9115, GSE27115, GSE29265, GSE33630, GSE35570, GSE50901, GSE53072, GSE53157, GSE58689 and GSE65144). Except for GSE53072, GSE53157 and GSE65144, the remaining chip arrays revealed significantly upregulated expression of CCND1 in the TC tissues compared with non-cancerous tissues $(\mathrm{P}<0.05$; Fig. 6B-N). The GSE53072, GSE53157 and GSE65144 chip arrays displayed non-significant trends towards upregulated expression of CCND1 in the TC tissues. AROC curve analysis of CCND1 was also performed and the results are shown in Fig. 7B-N. Then, CCND1 expression data from TCGA and GEO databases, which provided 897 TC and 193 non-cancerous tissues, were subjected to meta-analysis. The pooled SMD of CCND1 was $1.83(\mathrm{P}<0.001$; 95\% CI, 1.41-2.26; Fig. 8A) by the random effects model, and the test for heterogeneity was $\mathrm{P}<0.01\left(\mathrm{I}^{2}=79.9 \%\right)$. The sROC curve of the TCGA and GEO expression data is shown in Fig. 8B. The publication bias was evaluated by a funnel chart: Begg's test and Egger's test were 0.44 and 0.75 , respectively, which indicated that there was no conspicuous publication bias among the datasets (Fig. 8C and D). Furthermore, the AUC of the sROC was 0.91 (95\% CI, 0.89-0.94) and the combined sensitivity and specificity were 0.85 (95\% CI, 0.81-0.89) and 0.98 (95\% CI, 0.90-1.00), respectively. The results revealed that CCND1 may be a good biomarker to discriminate TC tissues from non-cancerous tissues.

Validation of the targeting regulatory relation between miR-193a-3p and CCND1. The putative miR-193a-3p binding site in CCND1 3' UTR was identified (Fig. 9A). The targeting regulatory association between miR-193a-3p and CCND1 was investigated in 293 cells following transfection with miR-193a-3p or miR-146b-5p mimics. The findings indicated that miR-193a-3p reduced the luciferase activity of the Wt CCND1 3' UTR, demonstrating a direct combination between CCND1 and miR-193a-3p (P<0.05; Fig. 9B-D). Furthermore, the luciferase activity of CCND1 3' UTR (Mut) 


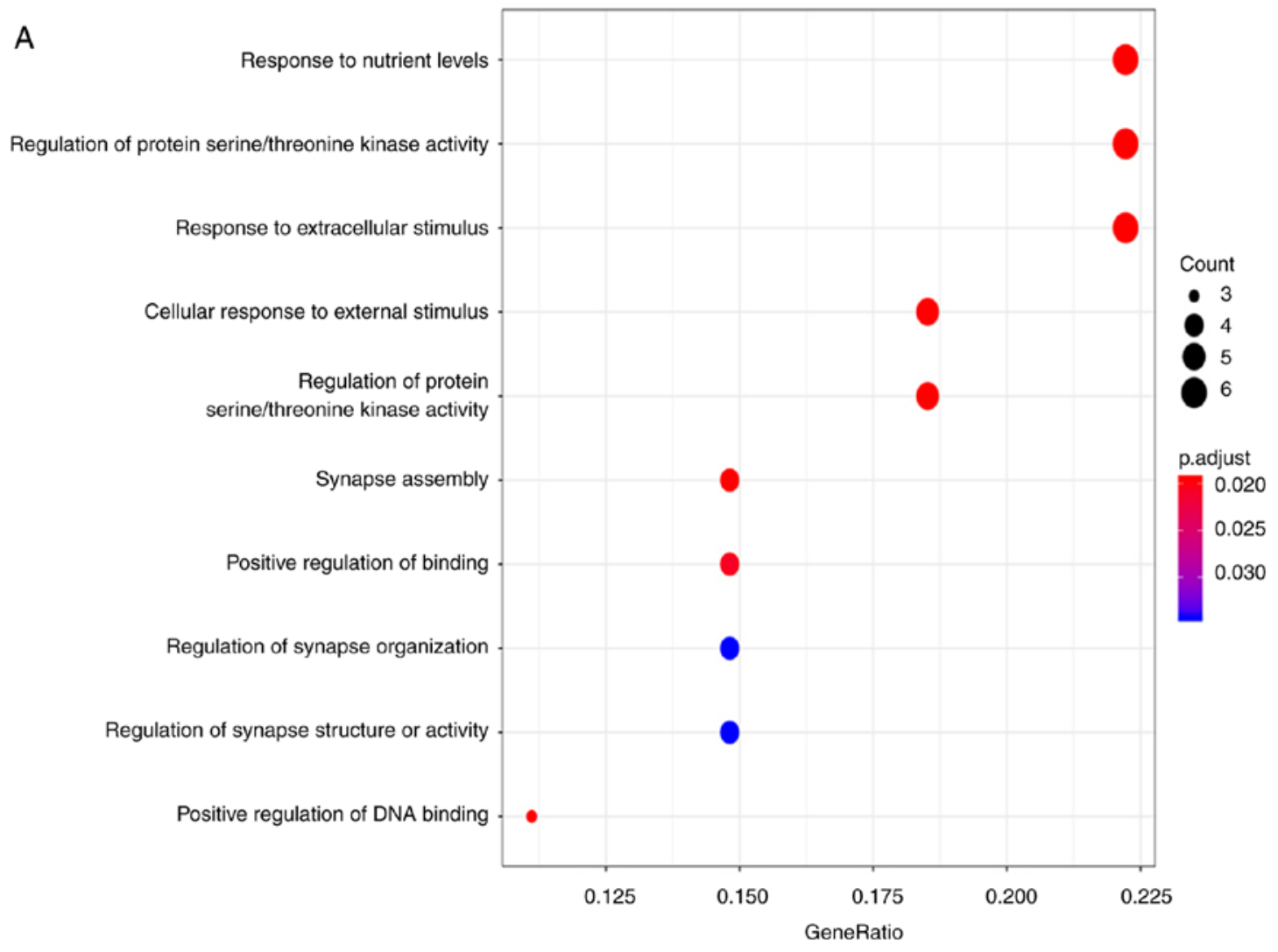

B

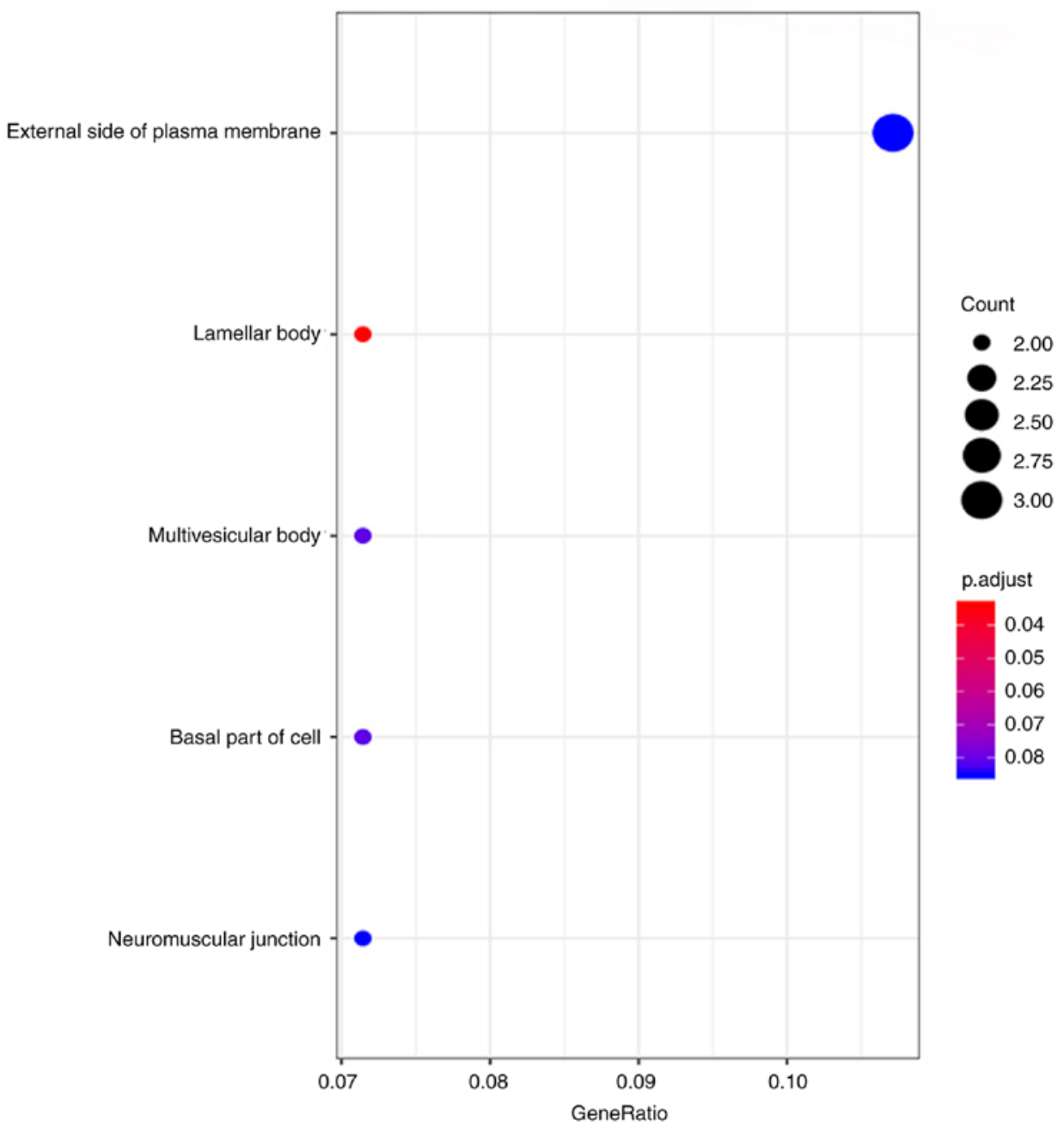

Figure 5. Gene Ontology and disease ontology analyses of microRNA-193a-3p target genes. (A) Biological processes. (B) Cellular components. 


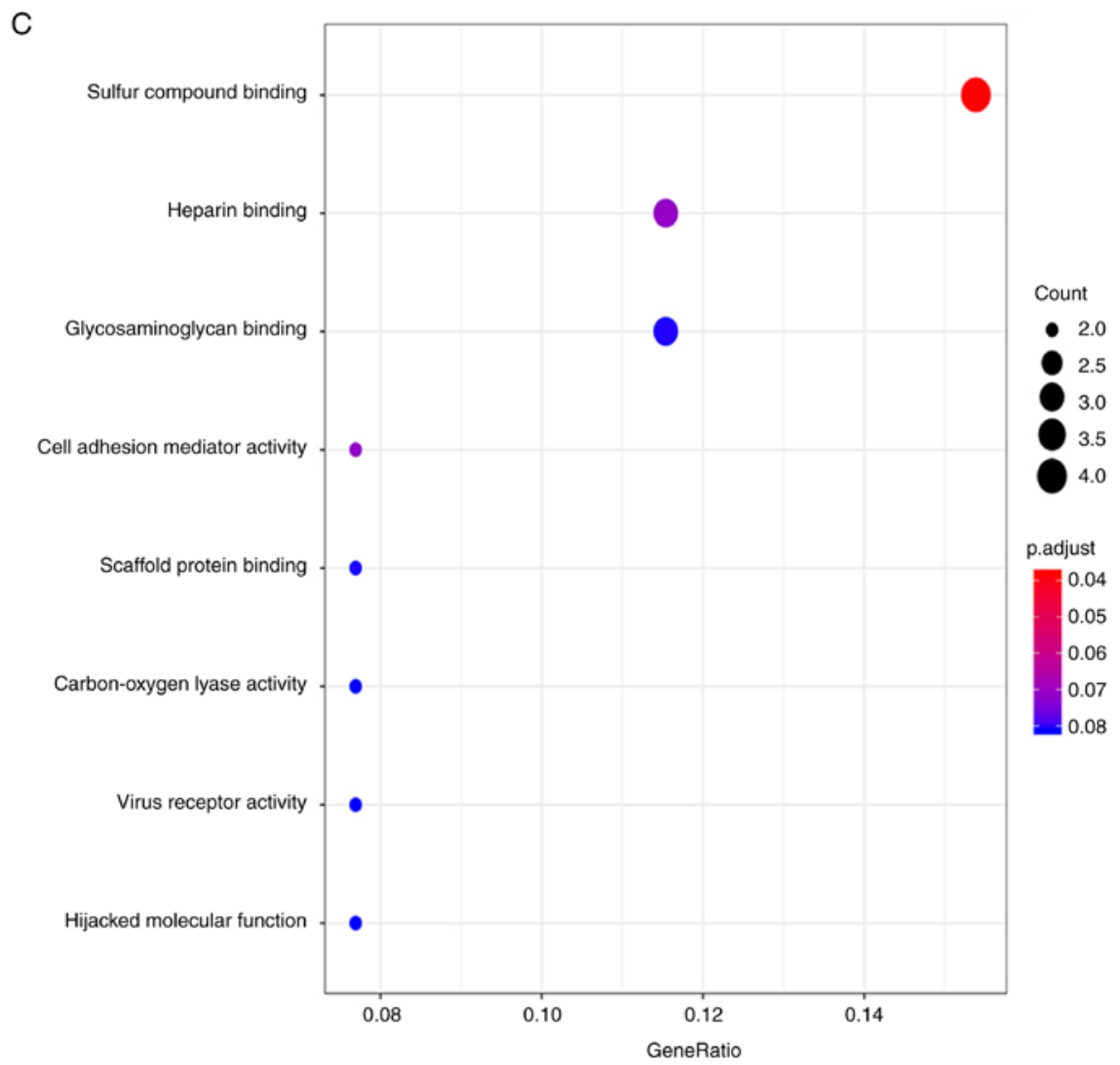

D

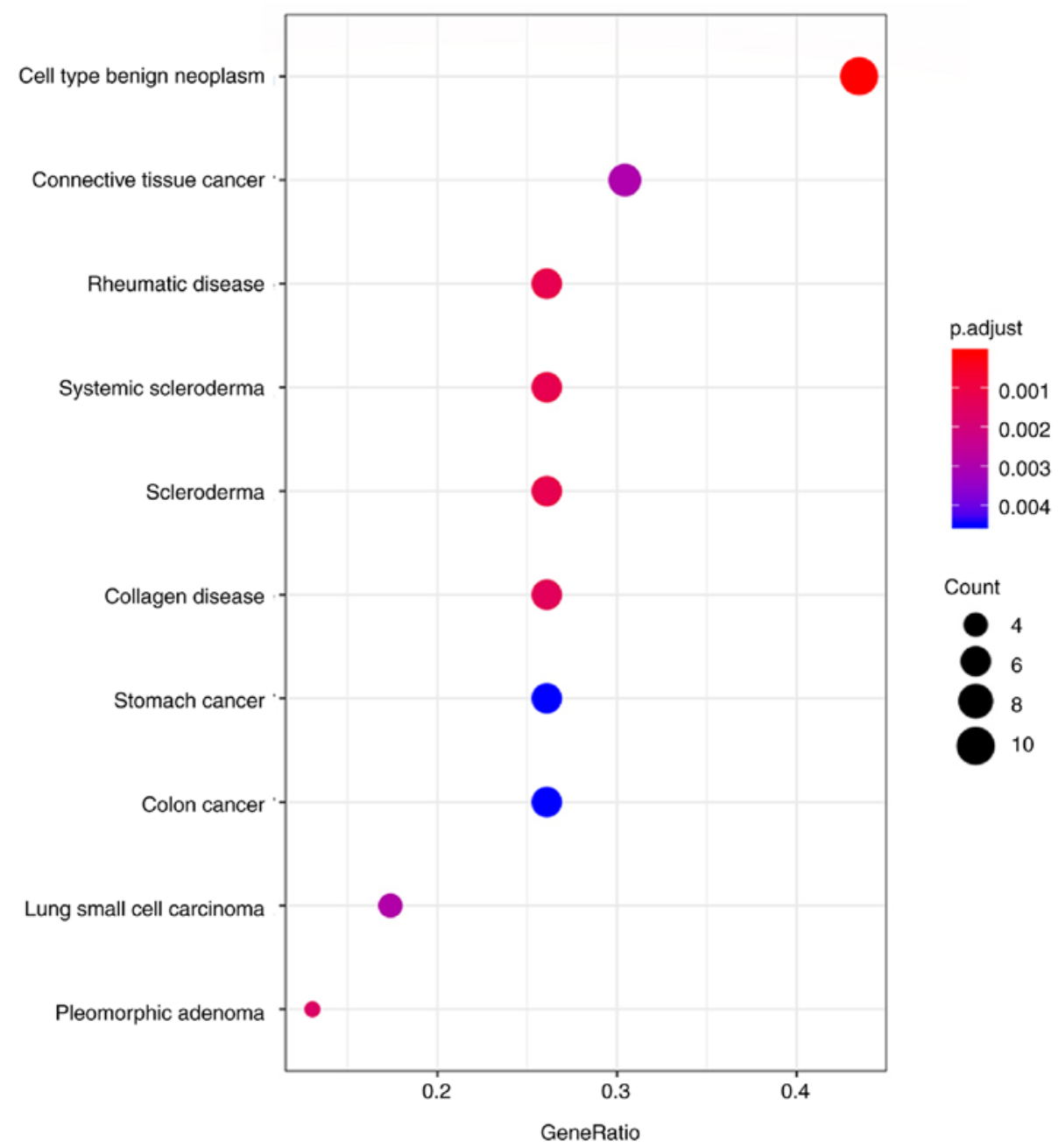

Figure 5. Cntinued. (C) Molecular functions. (D) Disease Ontology terms. 
Table III. Enrichment GO and disease ontology terms of the target genes of microRNA-193a-3p.

A, Biological processes

\begin{tabular}{llll}
\hline ID & \multicolumn{1}{c}{ Term } & Count & P-value \\
\hline GO:0031667 & Response to nutrient levels & 6 & $5.58 \times 10^{-5}$ \\
GO:0071900 & Regulation of protein serine/threonine kinase activity & 6 & $6.50 \times 10^{-5}$ \\
GO:0009991 & Response to extracellular stimulus & 6 & $8.06 \times 10^{-5}$ \\
\hline
\end{tabular}

B, Cellular component

\begin{tabular}{|c|c|c|c|}
\hline ID & Term & Count & P-value \\
\hline GO:0042599 & Lamellar body & 2 & $2.91 \times 10^{-4}$ \\
\hline GO:0005771 & Multivesicular body & 2 & $1.71 \times 10^{-3}$ \\
\hline GO:0045178 & Basal part of cell & 2 & $2.06 \times 10^{-3}$ \\
\hline
\end{tabular}

C, Molecular function

\begin{tabular}{llll}
\hline ID & \multicolumn{1}{c}{ Term } & Count & P-value \\
\hline GO:1901681 & Sulfur compound binding & 4 & $3.34 \times 10^{-4}$ \\
GO:0008201 & Heparin binding & 3 & $1.46 \times 10^{-3}$ \\
GO:0098631 & Cell adhesion mediator activity & 2 & $1.87 \times 10^{-3}$ \\
\hline
\end{tabular}

D, DO terms

\begin{tabular}{llrr}
\hline ID & \multicolumn{1}{c}{ Term } & Count & P-value \\
\hline DOID:0060084 & Cell type benign neoplasm & 10 & $2.79 \times 10^{-7}$ \\
DOID:1575 & Rheumatic disease & 6 & $1.12 \times 10^{-5}$ \\
DOID:418 & Systemic scleroderma & 6 & $1.12 \times 10^{-5}$ \\
\hline
\end{tabular}

GO, Gene Ontology; DO, Disease Ontology.

Table IV. General characteristics of included microarray and RNA sequencing datasets.

\begin{tabular}{|c|c|c|c|c|c|}
\hline Authors, year & Country & Dataset & Cancer group & Non-cancer groups & Refs. \\
\hline TCGA, 2018 & USA & TCGA & 513 & 59 & - \\
\hline Reyes et al, 2005 & USA & GSE3678 & 7 & 7 & Unpublished data \\
\hline Vasko et al, 2007 & USA & GSE6004 & 14 & 4 & $(57)$ \\
\hline Fontaine et al, 2008 & France & GSE6339 & 20 & 122 & $(58)$ \\
\hline Salvatore et al, 2007 & USA & GSE9115 & 15 & 4 & $(59)$ \\
\hline Giordano et al, 2006 & USA & GSE27155 & 78 & 21 & $(60)$ \\
\hline Tomas et al, 2011 & Belgium & GSE29265 & 29 & 20 & Unpublished data \\
\hline Dom et al, 2012 & Belgium & GSE33630 & 60 & 45 & $(61)$ \\
\hline Handkiewicz-Junak et al, 2016 & Poland & GSE35570 & 32 & 51 & $(62)$ \\
\hline Barros-Filho et al, 2015 & Brazil & GSE50901 & 61 & 4 & $(63)$ \\
\hline Pita et al, 2014 & Portugal & GSE53072 & 5 & 4 & $(64)$ \\
\hline Pita et al, 2009 & Portugal & GSE53157 & 24 & 3 & $(65)$ \\
\hline Rusinek et al, 2015 & Poland & GSE58689 & 27 & 18 & (66) \\
\hline von Roemeling et al, 2015 & USA & GSE65144 & 12 & 13 & (67) \\
\hline
\end{tabular}

TCGA, The Cancer Genome Atlas. 
A

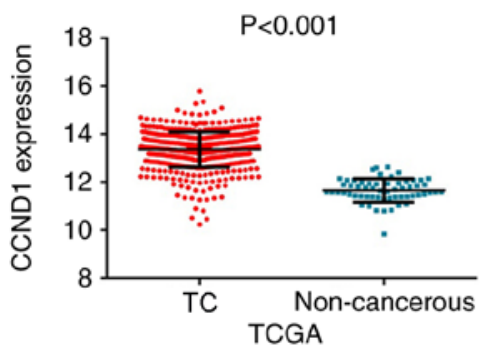

D

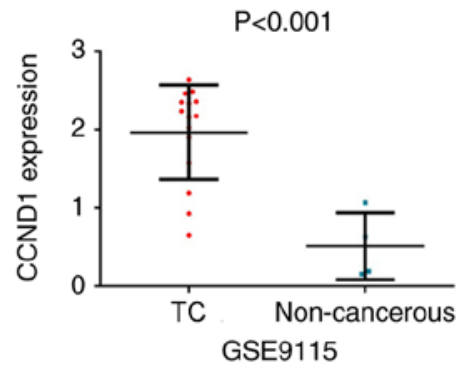

G

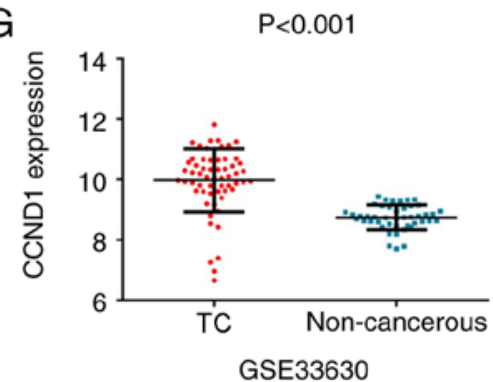

$J$

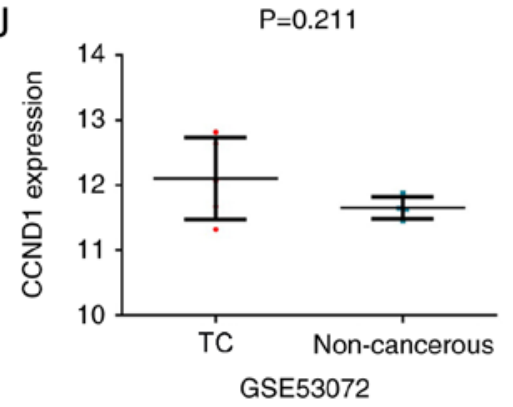

B

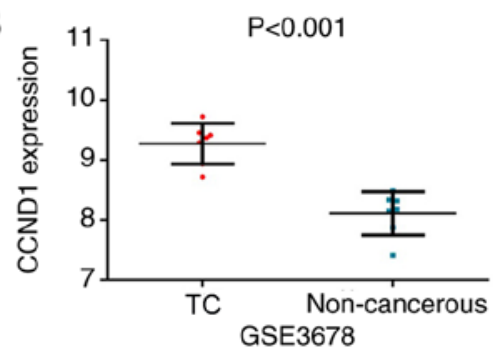

E

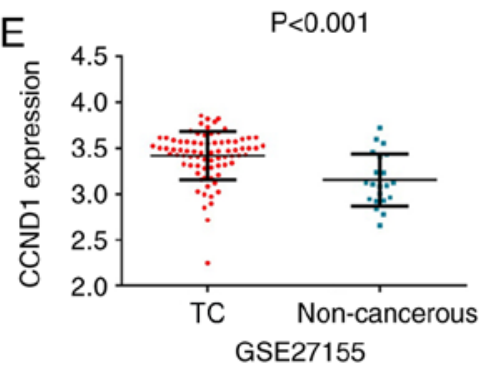

$\mathrm{H}$

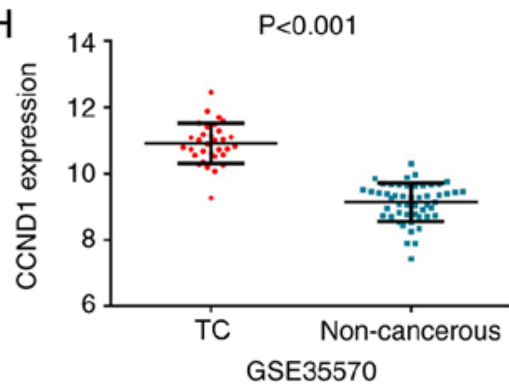

$\mathrm{K}$

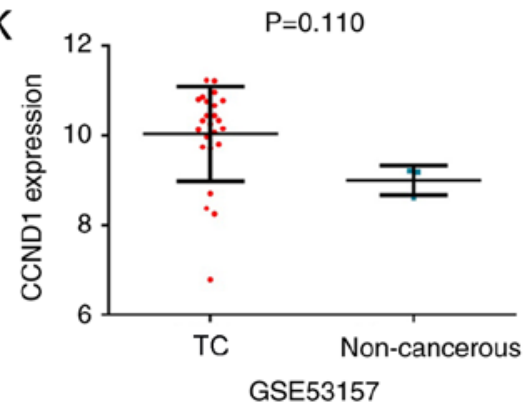

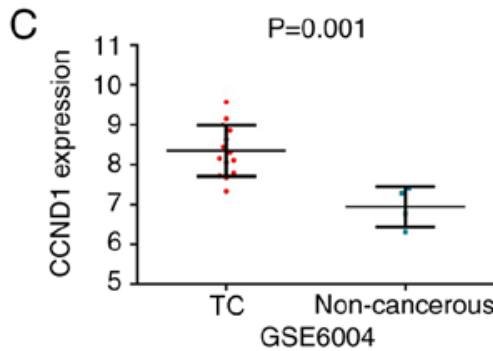

$\mathrm{F}$

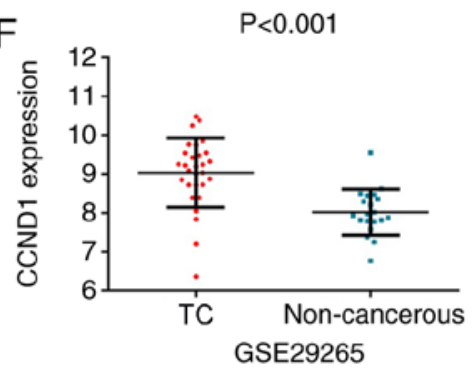

I

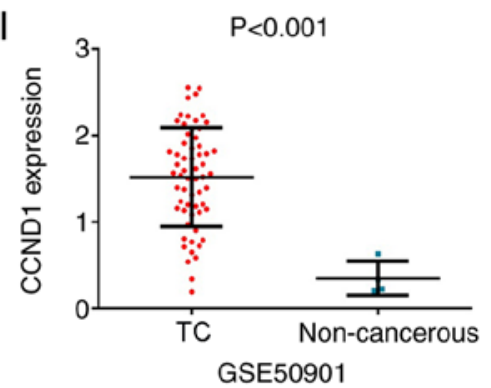

$\mathrm{L}$

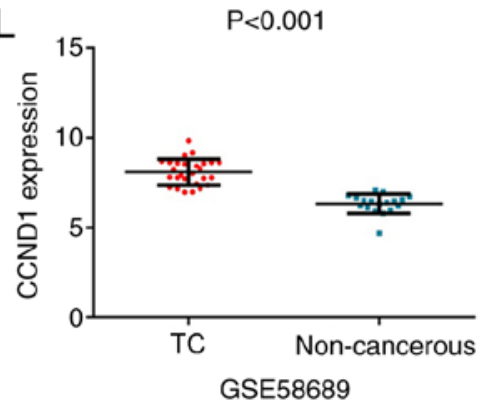

M

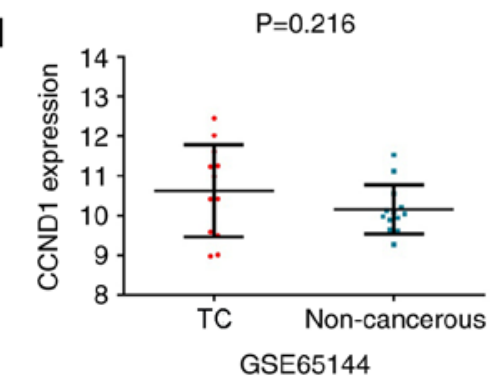

$\mathrm{N}$

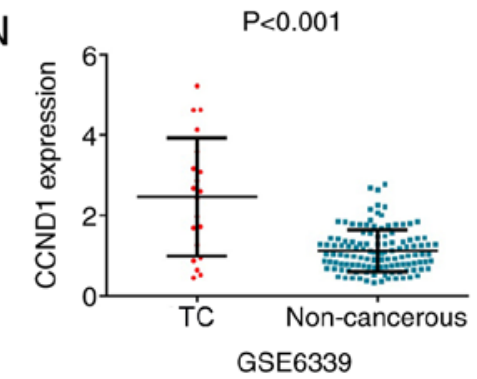

Figure 6. Upregulation of CCND1 in TC compared to non-cancerous tissues based on different databases. (A) TCGA, (B) GSE3678, (C) GSE6004, (D) GSE9115, (E) GSE27115, (F) GSE29265, (G) GSE33630, (H) GSE35570, (I) GSE50901, (J) GSE53072, (K) GSE53157, (L) GSE58689, (M) GSE65144 and (N) GSE6339. TC, thyroid cancer. CCND1, cyclin D1; TCGA, The Cancer Genome Atlas.

was not reduced in the mimic-transfected cells. miR-146-5p reduced the luciferase activity of the Wt TRAF6-3' UTR, indicating that the experiment and its results were reliable. Pearson correlation analysis was also conducted to evaluate the association between miR-193a-3p and CCND1; however, no linear correlation was observed between CCND1 and miR-193a-3p $(\mathrm{r}=-0.163$; Fig. 9E). The Human Protein Atlas database was utilized to explore the protein levels of CCND1. Consistent with the gene expression data, CCND1 protein expression was higher in TC than in non-cancerous tissues, as shown in Fig. 10. 

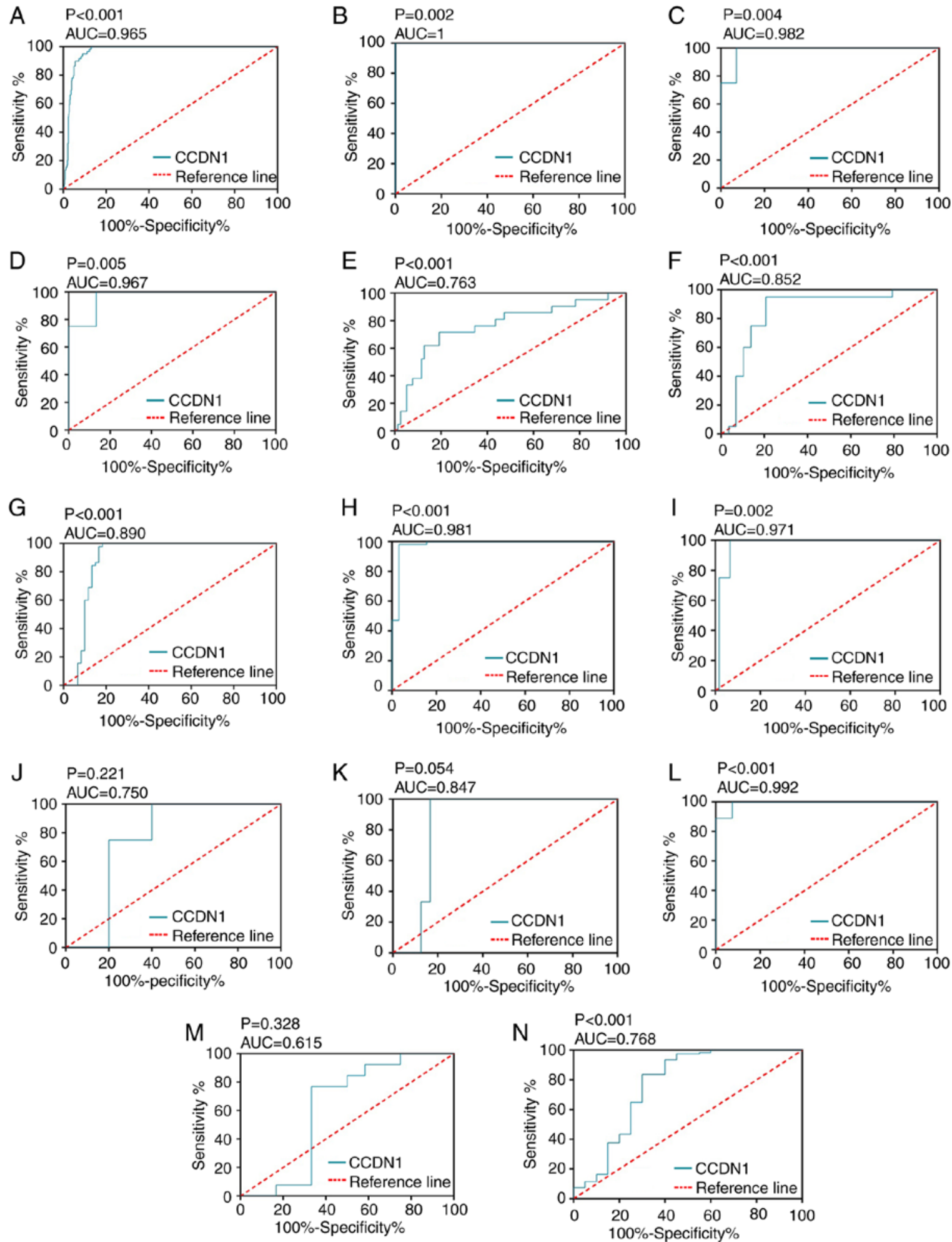

Figure 7. Receiver operating characteristic curves of CCND1 expression for differentiating thyroid cancer from non-cancerous thyroid tissues. (A) The Cancer Genome Atlas, (B) GSE3678, (C) GSE6004, (D) GSE9115, (E) GSE27115, (F) GSE29265, (G) GSE33630, (H) GSE35570, (I) GSE50901, (J) GSE53072, (K) GSE53157, (L) GSE58689, (M) GSE65144 and (N) GSE6339 datasets. CCND1, cyclin D1; AUC, area under curve.

\section{Discussion}

TC is a frequent endocrine malignancy of the endocrine system (72). The occurrence rate of TC has been increasing worldwide (73). The main therapy method of TC is thyroidectomy and radioiodine treatment (74). To the best of the authors' knowledge, there is currently no effective drug for the treatment for TC. There is increasing evidence that abnormal miRNA expression levels give rise to TC tumorigenesis and progression. Prior studies have demonstrated that various miRNAs may aid in the diagnosis of TC and help formulate more individualized therapeutic management strategies (75-78). miR-193a-3p 
Table V. Clinical parameters and CCND1 expression in TC from The Cancer Genome Atlas.

\begin{tabular}{|c|c|c|c|c|}
\hline \multirow[b]{2}{*}{ Clinicopathological feature } & \multirow[b]{2}{*}{ Cases } & \multicolumn{3}{|c|}{ CCND1 expression } \\
\hline & & Mean \pm SD & $\mathrm{t} / \mathrm{F}$ value & P-value \\
\hline \multicolumn{5}{|l|}{ Tissue } \\
\hline Non-cancerous tissues & 59 & $11.66 \pm 0.49$ & \multirow[t]{2}{*}{-23.800} & \multirow[t]{2}{*}{$<0.001$} \\
\hline $\mathrm{TC}$ & 513 & $13.36 \pm 0.74$ & & \\
\hline \multicolumn{5}{|l|}{ Sex } \\
\hline Male & 136 & $13.33 \pm 0.80$ & \multirow[t]{2}{*}{-0.574} & \multirow[t]{2}{*}{0.566} \\
\hline Female & 369 & $13.37 \pm 0.72$ & & \\
\hline \multicolumn{5}{|l|}{ Pathologic tumor grade } \\
\hline Stage I-II & 336 & $13.33 \pm 0.77$ & \multirow[t]{2}{*}{-1.269} & \multirow[t]{2}{*}{0.205} \\
\hline Stage III-IV & 167 & $13.42 \pm 0.68$ & & \\
\hline \multicolumn{5}{|l|}{ Age } \\
\hline$<60$ & 385 & $13.38 \pm 0.73$ & \multirow[t]{2}{*}{1.114} & \multirow[t]{2}{*}{0.266} \\
\hline$\geq 60$ & 120 & $13.30 \pm 0.78$ & & \\
\hline \multicolumn{5}{|l|}{ TNM stage } \\
\hline $\mathrm{T} 1-\mathrm{T} 2$ & 309 & $13.33 \pm 0.74$ & \multirow[t]{2}{*}{-1.148} & \multirow[t]{2}{*}{0.251} \\
\hline $\mathrm{T} 3-\mathrm{T} 4$ & 194 & $13.41 \pm 0.74$ & & \\
\hline \multicolumn{5}{|l|}{ Pathological lymph node } \\
\hline No & 230 & $13.32 \pm 0.84$ & \multirow[t]{2}{*}{-1.849} & \multirow[t]{2}{*}{0.065} \\
\hline Yes & 225 & $13.44 \pm 0.55$ & & \\
\hline \multicolumn{5}{|l|}{ Metastasis } \\
\hline No & 282 & $13.40 \pm 0.63$ & \multirow[t]{2}{*}{-0.662} & \multirow[t]{2}{*}{0.508} \\
\hline Yes & 9 & $13.55 \pm 0.71$ & & \\
\hline \multicolumn{5}{|l|}{ Ethnicity } \\
\hline Caucasian & 334 & $13.38 \pm 0.69$ & \multirow[t]{3}{*}{1.278} & \multirow[t]{3}{*}{0.280} \\
\hline African-American & 27 & $13.58 \pm 0.43$ & & \\
\hline Asian & 51 & $13.45 \pm 0.73$ & & \\
\hline
\end{tabular}

CCND1, cyclin D1; TC, thyroid cancer.

is involved in certain biological processes, including apoptosis, proliferation, invasion, metastasis and migration (79). A number of studies have demonstrated that miR-193a-3p is expressed at low levels in different human cancers, including colon cancer $(80)$, lung cancer $(81,82)$, breast cancer $(28)$ and malignant pleural mesothelioma (83). Nevertheless, the expression levels, as well as its mechanisms, in TC remain to be elucidated. Hence, a comprehensive meta-analysis was conducted to evaluate clinical valuation of miR-193a-3p in TC. GO, KEGG pathway and PPI analyses were performed to search for the latent biological mechanisms of miR-193a-3p in TC. The results indicated that CCND1 served as the most important hub gene. Subsequently, the relationship between miR-193a-3p and CCND1 was validated through a dual luciferase assay.

To the best of the authors' knowledge, the present study is the first to survey the expression levels and clinical value of miR-193a-3p in TC by extracting expression data from TCGA, GEO and ArrayExpress. The present study primarily scrutinized the miR-193a-3p expression between 510 TC and 59 non-cancerous thyroid samples using TCGA database. The outcomes revealed that miR-193a-3p had low expression in TC. Subsequently, the GEO database and ArrayExpress were used to validate miR-193a-3p expression in TC. A total of 2 of the 4 chip arrays also revealed that a low miR-193a-3p expression is significant in TC. Although the remaining 2 chip arrays did not display a significant downregulation in TC tissues, they indicated the downregulated expression tendency of miR-193a-3p in TC. According to the results of the present study, miR-193a-3p may provide a biomarker to test the occurrence and development of TC. However, in the study by Santarpia et al (31), the result was the opposite: miR-193a-3p was overexpressed in TC compared with non-cancerous groups. The observation of overexpressed miR-193a-3p may be caused by the small sample size of the study. That significantly downregulated miR-193a-3p in TC was observed in the present study was credible due to the quantity of sample data that was obtained from several public databases. Analysis of the sROC in miR-193a-3p from TCGA, GEO and ArrayExpress databases demonstrated that the expression levels may have a moderate ability to differentiate between TC patients and non-cancerous controls with an AUC of 0.80. At the same 


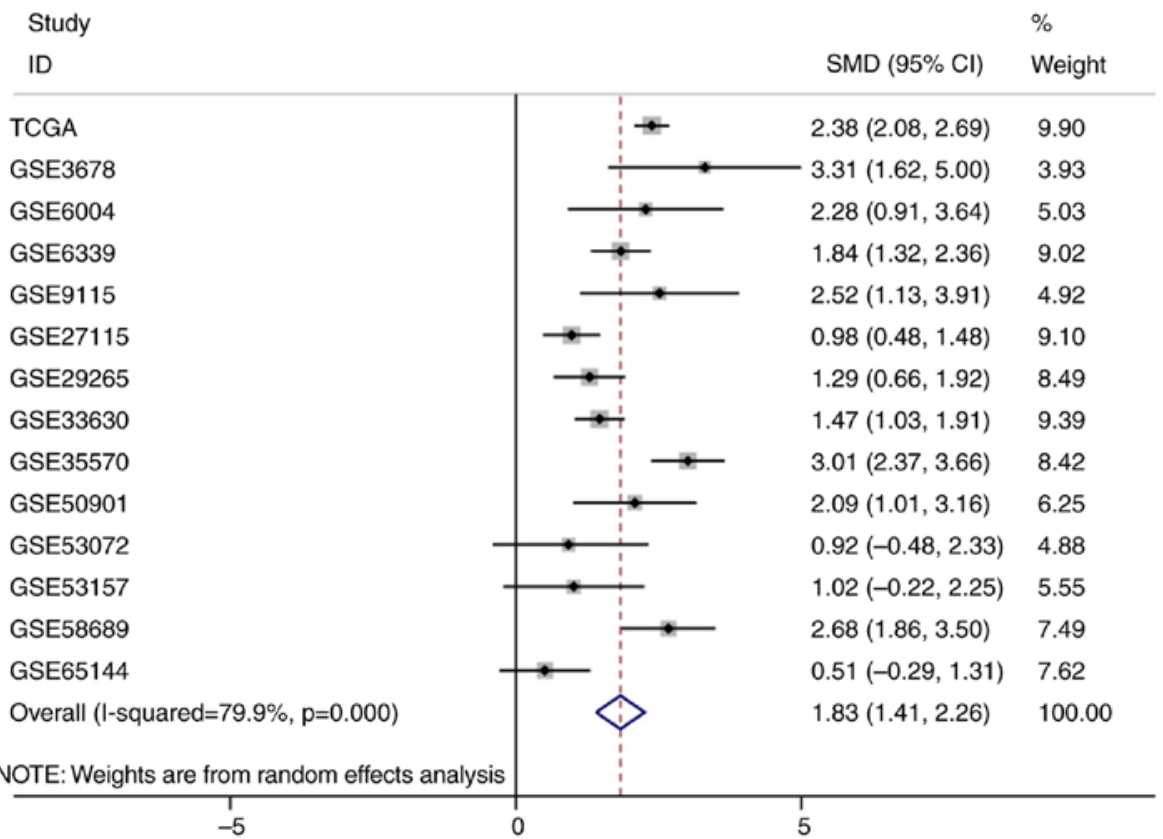

B

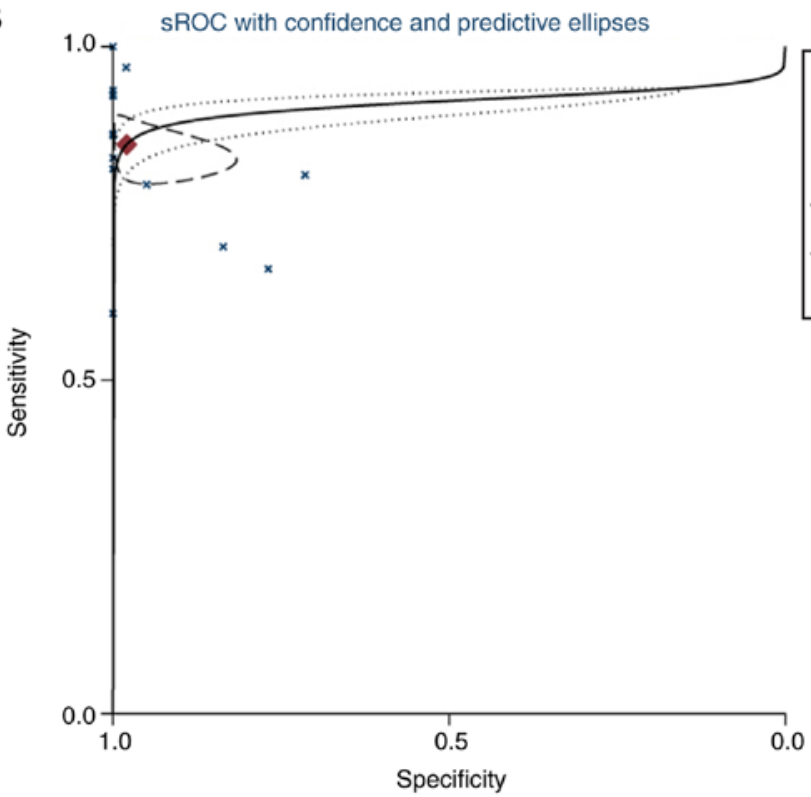

C Begg's funnel plot with pseudo $95 \%$ confidence limits

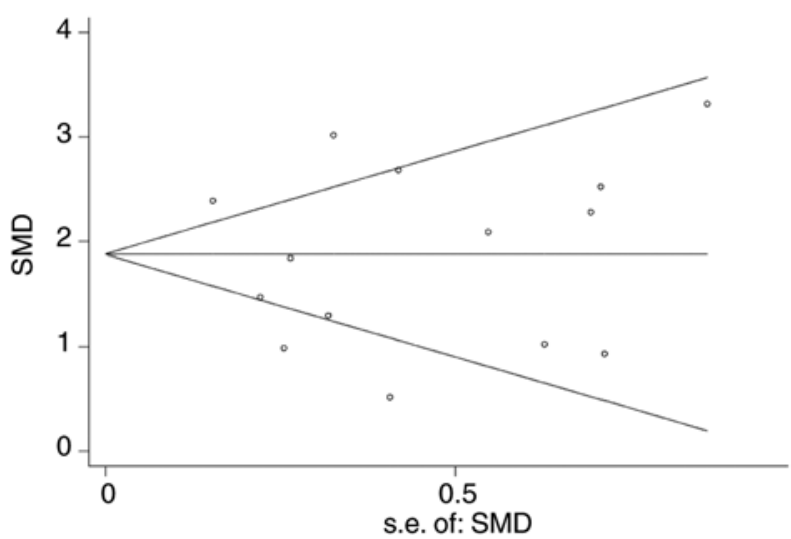

D Egger's publication bias plot

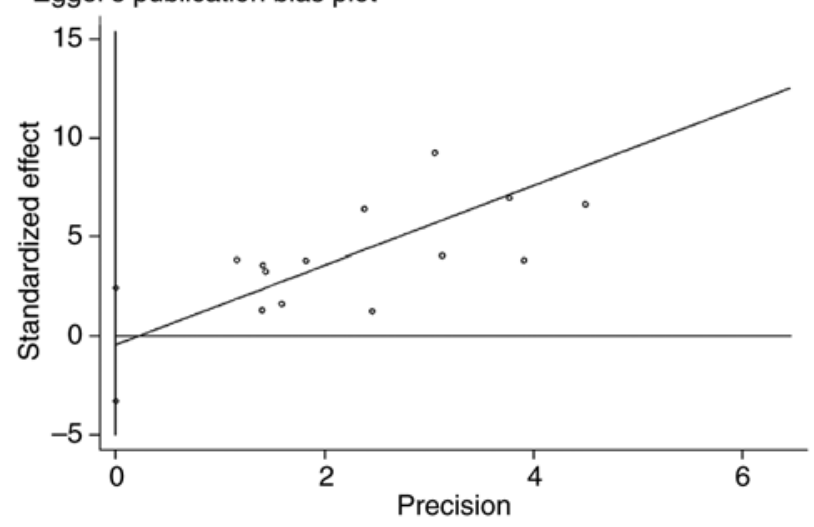

Figure 8. Meta-analysis of CCND1 from public databases. (A) Forest plot curve of the meta-analysis for evaluating CCND1 expression between TC and non-cancerous tissues, the merged SMD was 1.83 ( $\mathrm{P}<0.001$; 95\% CI, 1.41-2.26). (B) sROC of CCND1 in TC from TCGA and GEO databases was 0.91 (95\% CI, 0.89-0.94), which demonstrated that CCND1 probably plays crucial role in distinguishing TC from non-cancerous tissues. The pooled sensitivity and specificity at 0.85 (95\% CI, 0.81-0.89) and 0.98 (95\% CI, 0.90-1.00), respectively. (C) Begg's test. (D) Egger's test. CCND1, cyclin D1; TC, thyroid cancer; SMD, standard mean difference; sROC, summary receiver operating characteristic; TCGA, The Cancer Genome Atlas; GEO, Gene Expression Omnibus. 
A

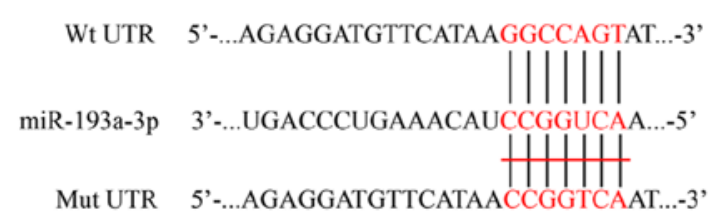

C

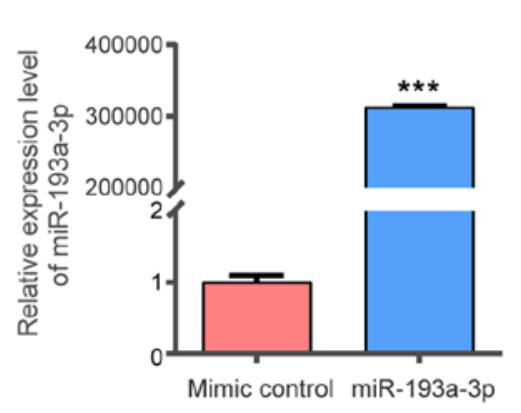

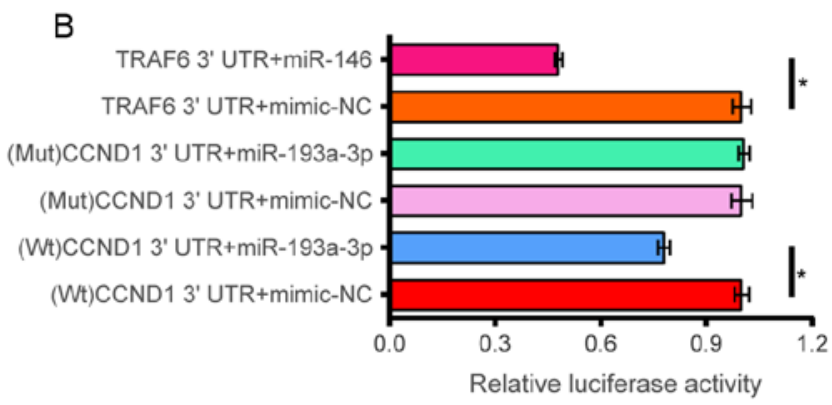

$\mathrm{E}$

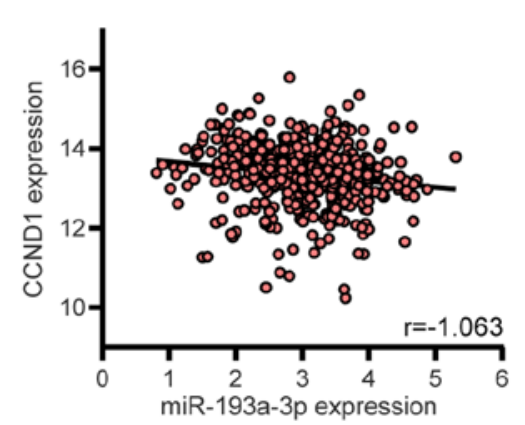

Figure 9. Dual luciferase assay. (A) miR-193a-3p binding site in the 3' UTR region of CCND1. (B) After co-transfection into 293 cells, miR-193a-3p reduced the luciferase activity of the CCND1 3' UTR $(\mathrm{P}<0.05)$. TRAF6 3' UTR + mimic-NC and TRAF6 3' UTR + miR-146 were used as positive control. The x-axis represented the relative fluorescence value. The y-axis represented different test groups. " $\mathrm{P}<0.05$. Expression of (C) miR-193a-3p and (D) miR-146 in 293 cells following transfection with mimic. ${ }^{* *} \mathrm{P}<0.01,{ }^{* * *} \mathrm{P}<0.001$ vs. mimic control. (E) Pearson's correlation analysis between miR-193a-3p expression levels and CCND1 levels from TCGA ( $r=-0.163)$. miR, microRNA. miR, microRNA; CCND1, cyclin D1; TNFR6, tumor necrosis factor receptor associated factor 6; UTR, untranslated region; Wt, wild-type; Mut, mutant; NC, negative control.
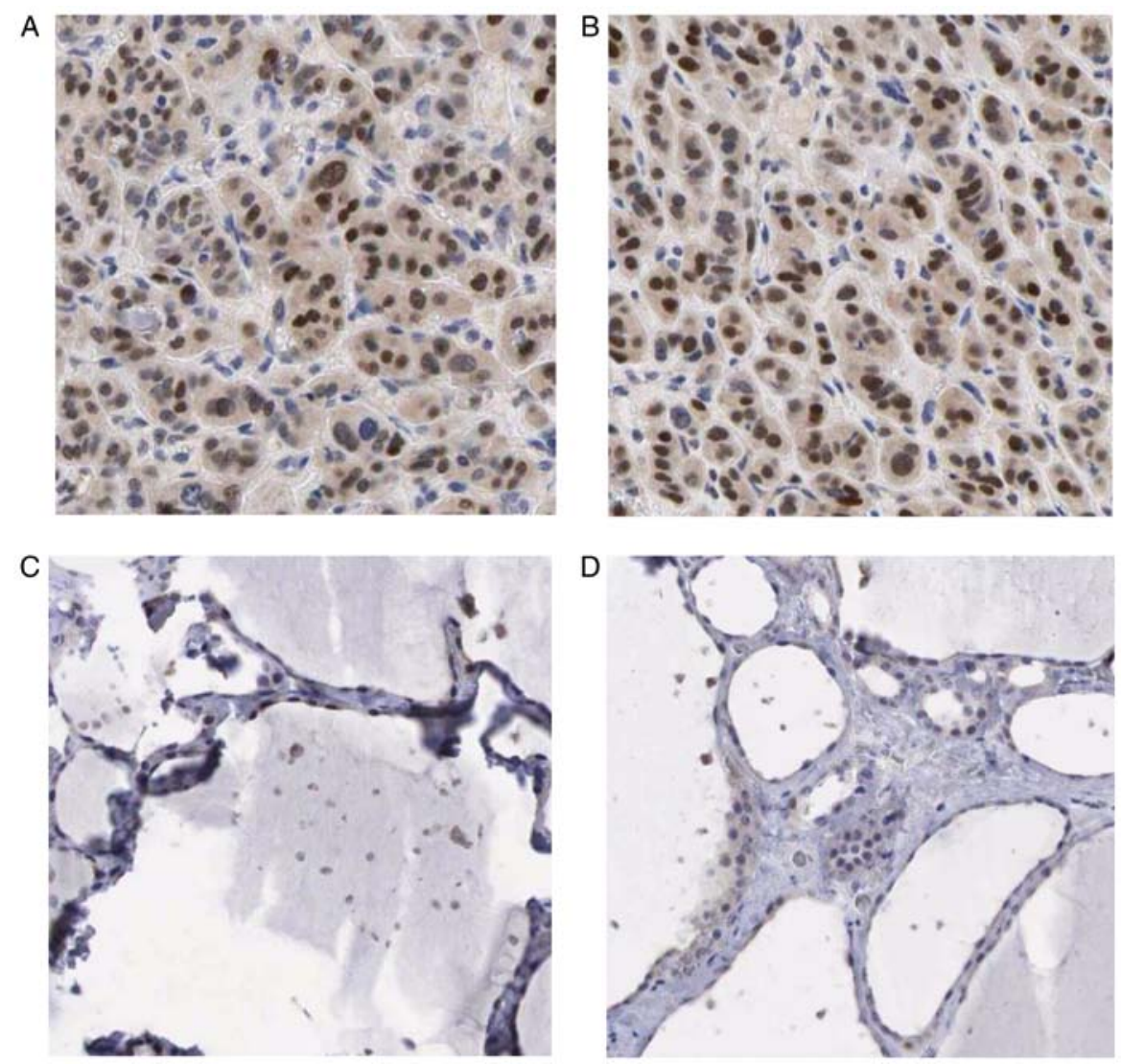

Figure 10. Immunohistochemical staining for CCND1 in thyroid cancer tissues from The Human Protein Atlas. (A and B) CCND1 expression was clearly observed in follicular adenoma carcinoma tissues (version 19.3; https://www.proteinatlas.org/ENSG00000110092-CCND1/pathology/ thyroid+cancer\#img). (C and D) CCND1 expression was not detected in normal thyroid tissues. Magnification, x200 (version 19.3; https://www.proteinatlas. org/ENSG00000110092-CCND1/tissue/Thyroid+gland\#img). CCND1, cyclin D1. 
time, its sensitivity and specificity were 0.69 and 0.82 , respectively. Additionally, downregulated miR-193a-3p was association with age, metastasis and sex in TCGA. Although the present study identified that downregulated miR-193a-3p was associated with age, metastasis and sex; how these factors regulate TC cells requires further investigation by in vivo and/or in vitro analysis.

At present, several studies have noted that abnormal miR-193a-3p expression has a pivotal effect on cell biological processes and leads to numerous pathological conditions $(79,80,84)$. For example, Pekow et al revealed that miR-193a-3p is a crucial neoplasm inhibitor in colon carcinoma and facilitates the progression of cancer (80). Lin et al (84). demonstrated that low miR-193a-3p expression is related to a poor disease outcome in colorectal carcinoma. In addition, miR-193a-3p suppresses cell multiplication in prostatic carcinoma by targeting CCND1 (85). However, little research has been performed into the molecular mechanisms of miR-193a-3p in TC. To further study the underlying mechanisms regarding the regulation of miR-193a-3p in TC, the present study conducted a bioinformatics analysis of the candidate target genes of miR-193a-3p. A total of 28 candidate target genes of miR-193a-3p were gathered and GO analysis was performed, which indicated that the candidate target genes markedly assembled in 'response to nutrient levels' for $\mathrm{BP}$, 'lamellar body' for CC and 'sulfur compound binding' for MF. GO analysis revealed that miR-193a-3p target genes were mainly concentrated in 'cell adhesion', which exerts an oncogenic role in the progression of TC. In addition, PPI analysis was performed, and it was found that CCND1 was situated at the core of the PPI network. CCND1 had the most connections among proteins, which may manifest in the way the gene contributes to TC.

A previous study by Li et al (35) reported that CCND1 plays a key role in facilitating migration and tumor metastasis. Liang and Sun (86) reported that an upregulation of CCND1 in thyroid cancer tissues is markedly associated with the clinical stage of TC, indicating that it could be considered as a biomarker in clinical settings. A study by Jeon et al (87) showed that CCND1 is clearly overexpressed in TC, and may be involved in the occurrence and development of the disease. Lamba Saini et al (88) demonstrated that CCND1 and its variants are significantly overexpressed in $\mathrm{TC}$, and that it should serve as a diagnostic marker for TC. Investigating the mechanism of CCND1 expression levels is crucial due to the broader roles of CCND1 in TC tissues. From the present study, the results calculated from the TCGA database demonstrated that CCND1 exhibited a notably low expression in TC compared with non-cancerous tissues. However, differential expression of CCND1 was not obtained for the clinical parameters analyzed. The present study also included 13 microarrays from the GEO database. In addition to GSE53072, GSE53157, GSE6339 and GSE65144, other chip arrays all displayed a significant upregulation of CCND1 expression in TC compared to non-cancerous tissues. Although the remaining 4 chip arrays did not show statistical significance, they all showed an increasing trend in CCND1 expression levels in TC. From the sROC analysis of miR-193a-3p, the AUC was 0.91. The results showed that CCND1 may have a high value for differentiating TC from non-cancerous tissue and may serve as a prognostic biomarker in TC.

The study by Liu et al (85) identified that miR-193a-3p acts as a key component inhibiting prostate cancer, which could induce G1-phase arrest by targeting CCND1. Chou et al (25) demonstrated that miR-193a-3p suppresses the aggression and progression of gastric cancer by targeting CCND1 expression. Tsai et al (89) also demonstrated that miR-193a-3p may serve as an underlying neoplasm inhibitor in breast carcinoma, which could inhibit cell growth by suppressing CCND1. To this end, the present study performed a dual luciferase assay, and a regulatory association between miR-193a-3p and CCND1 was validated. In summary, the findings of the present study revealed that miR-193a-3p may regulate CCND1 expression to affect the tumor cell cycle, cell proliferation, differentiation and metabolism. Nevertheless, further research is required to test this hypothesis.

However, in the present study, some limitations should be acknowledged. First, it lacks further in vitro and in vivo testing, such as reverse transcription-quantitative PCR. These methods are pivotal to enhancing the understanding of how miR-193a-3p works in relation to CCND1 in TC. Second, the present study only focused on the analysis of differential miRNAs in tissues. Other samples, such as blood, should also be assessed.

In summary, the present study gathered a great amount of data from TCGA, GEO, and ArrayExpress databases, and validated the clinical value of miR-193a-3p and CCND1. The results showed that miR-193a-3p expression was evidently downregulated in TC, while CCND1 expression was markedly upregulated. These results may help to diagnose TC and predict the prognosis of TC when thyroid tissues are obtained from a fine needle biopsy. Bioinformatics analysis was next performed to clarify the molecular mechanisms of miR-193a-3p. In light of the bioinformatics analysis, CCND1 was regarded as the most important hub gene in TC. Subsequently, a dual luciferase assay was performed to corroborate the targeting regulatory association between miR-193a-3p and CCND1. The results demonstrated that miR-193a-3p markedly contributes to TC via particular pathways, and both miR-193a-3p and CCND1 may serve as potential biological markers of TC.

\section{Acknowledgements}

Not applicable.

\section{Funding}

This study was supported by Fund of National Natural Science Foundation of China (grant nos. NSFC81060202 and NSFC81260222), the Fund of Guangxi Key R\&D Project Plan (grant no. AB17195020) and the Future Academic Star of Guangxi Medical University (grant nos. WLXSZX19050 and WLXSZX19055).

\section{Availability of data and materials}

The datasets used and/or analyzed during the current study are available from the corresponding author on reasonable request. 


\section{Authors' contributions}

YYP, HY, XJL and RW contributed to the design of the study; XJL and RW wrote the manuscript; DYW, PL, DHP, LJZ and $\mathrm{YH}$ collected the data, performed the statistical analysis and interpreted the data; and LS, YYQ, YHL, JNL, JLY, QQL, JW and JM contributed to the data collection and statistical analysis. All authors read and approved the final manuscript.

\section{Ethics approval and consent to participate}

Not applicable.

\section{Patient consent for publication}

Not applicable.

\section{Competing interests}

The authors declare that they have no competing interests.

\section{References}

1. Konturek A, Barczyński M, Stopa M and Nowak W: Trends in Prevalence of Thyroid Cancer Over Three Decades: A Retrospective Cohort Study of 17,526 Surgical Patients. World J Surg 40: 538-544, 2016.

2. van der Zwan JM, Mallone S, van Dijk B, Bielska-Lasota M, Otter R, Foschi R, Baudin E and Links TP; RARECARE WG: Carcinoma of endocrine organs: Results of the RARECARE project. Eur J Cancer 48: 1923-1931, 2012.

3. Miller KD, Goding Sauer A, Ortiz AP, Fedewa SA, Pinheiro PS, Tortolero-Luna G, Martinez-Tyson D, Jemal A and Siegel RL: Cancer Statistics for Hispanics/Latinos, 2018. CA Cancer J Clin 68: 425-445, 2018

4. Lin P, Guo YN, Shi L, Li XJ, Yang H, He Y, Li Q, Dang YW, Wei KL and Chen G: Development of a prognostic index based on an immunogenomic landscape analysis of papillary thyroid cancer. Aging (Albany NY) 11: 480-500, 2019.

5. Zarkesh M, Zadeh-Vakili A, Akbarzadeh M, Fanaei SA, Hedayati $M$ and Azizi F: The role of matrix metalloproteinase-9 as a prognostic biomarker in papillary thyroid cancer. BMC Cancer 18: 1199, 2018.

6. Siegel RL, Miller KD and Jemal A: Cancer statistics, 2018. CA Cancer J Clin 68: 7-30, 2018.

7. Carling T and Udelsman R: Thyroid cancer. Annu Rev Med 65: $125-137,2014$.

8. Lin P, He Y, Wen DY, Li XJ, Zeng JJ, Mo WJ, Li Q, Peng JB, $\mathrm{Wu} Y \mathrm{YQ}, \mathrm{Pan} \mathrm{DH}$, et al: Comprehensive analysis of the clinical significance and prospective molecular mechanisms of differentially expressed autophagy-related genes in thyroid cancer. Int $\mathrm{J}$ Oncol 53: 603-619, 2018.

9. Liu C, Su C, Chen Y and Li G: miR-144-3p promotes the tumor growth and metastasis of papillary thyroid carcinoma by targeting paired box gene 8. Cancer Cell Int 18: 54, 2018.

10. Acquaviva G, Visani M, Repaci A, Rhoden KJ, de Biase D, Pession A and Giovanni T: Molecular pathology of thyroid tumours of follicular cells: A review of genetic alterations and their clinicopathological relevance. Histopathology 72: 6-31, 2018.

11. Riesco-Eizaguirre G and Santisteban P: Molecular biology of thyroid cancer initiation. Clin Transl Oncol 9: 686-693, 2007.

12. DeLellis RA: Pathology and genetics of thyroid carcinoma. J Surg Oncol 94: 662-669, 2006.

13. Liu T, You X, Sui J, Shen B, Zhang Y, Zhang XM, Yang S, Yao YZ, Yang F, Yin LH, et al: Prognostic value of a two-microRNA signature for papillary thyroid cancer and a bioinformatic analysis of their possible functions. J Cell Biochem: Nov 2, 2018 (Epub ahead of print). doi: 10.1002/jcb.27993 2018.

14. Wang X, Huang S, Li X, Jiang D, Yu H, Wu Q, Gao C and Wu Z: A potential biomarker hsa-miR-200a-5p distinguishing between benign thyroid tumors with papillary hyperplasia and papillary thyroid carcinoma. PLoS One 13: e0200290, 2018.
15. Vuong HG, Altibi AM, Abdelhamid AH, Ngoc PU, Quan VD, Tantawi MY, Elfil M, Vu TL, Elgebaly A, Oishi N, et al: The changing characteristics and molecular profiles of papillary thyroid carcinoma over time: A systematic review. Oncotarget 8: 10637-10649, 2017

16. Tricoli JV and Jacobson JW: MicroRNA: Potential for Cancer Detection, Diagnosis, and Prognosis. Cancer Res 67: 4553-4555, 2007.

17. Boufraqech M, Klubo-Gwiezdzinska $J$ and Kebebew E: MicroRNAs in the thyroid. Best Pract Res Clin Endocrinol Metab 30: 603-619, 2016.

18. Macha MA, Seshacharyulu P, Krishn SR, Pai P, Rachagani S, Jain M and Batra SK: MicroRNAs (miRNAs) as biomarker(s) for prognosis and diagnosis of gastrointestinal (GI) cancers. Curr Pharm Des 20: 5287-5297, 2014.

19. Parvex P: Are microRNA potential biomarkers in children with idiopathic nephrotic syndrome? EBioMedicine 39: 27-28, 2019.

20. Farazi TA, Spitzer JI, Morozov P and Tuschl T: miRNAs in human cancer. J Pathol 223: 102-115, 2011.

21. Li Z, Yu X, Shen J, Law PT, Chan MT and Wu WK: MicroRNA expression and its implications for diagnosis and therapy of gallbladder cancer. Oncotarget 6: 13914-13921, 2015.

22. Li L, Peng M, Xue W, Fan Z, Wang T, Lian J, Zhai Y, Lian W, Qin D and Zhao J: Integrated analysis of dysregulated long non-coding RNAs/microRNAs/mRNAs in metastasis of lung adenocarcinoma. J Transl Med 16: 372, 2018.

23. Ma Y and Sun Y: miR-29a-3p inhibits growth, proliferation, and invasion of papillary thyroid carcinoma by suppressing NF- $x \mathrm{~B}$ signaling via direct targeting of OTUB2. Cancer Manag Res 11: 13-23, 2018.

24. Liu Y,RenF,Luo Y,Rong M,Chen G and Dang Y: Down-Regulation of miR-193a-3p Dictates Deterioration of HCC: A Clinical Real-Time qRT-PCR Study. Med Sci Monit 21: 2352-2360, 2015.

25. Chou NH, Lo YH, Wang KC, Kang CH, Tsai CY and Tsai KW: miR-193a-5p and -3p Play a Distinct Role in Gastric Cancer: miR-193a-3p Suppresses Gastric Cancer Cell Growth by Targeting ETS1 and CCND1. Anticancer Res 38: 3309-3318, 2018.

26. Huang Y, Luo H, Li F, Yang Y, Ou G, Ye X and Li N: LINC00152 down-regulated miR-193a-3p to enhance MCL1 expression and promote gastric cancer cells proliferation. Biosci Rep 38: BSR20171607, 2018.

27. Deng W, Yan M, Yu T, Ge H, Lin H, Li J, Liu Y, Geng Q, Zhu M, Liu L, et al: Quantitative proteomic analysis of the metastasis-inhibitory mechanism of miR-193a-3p in non-small cell lung cancer. Cell Physiol Biochem 35: 1677-1688, 2015.

28. Yu M, Liu Z, Liu Y, Zhou X, Sun F, Liu Y, Li L, Hua S, Zhao Y, Gao H, et al: PTP1B markedly promotes breast cancer progression and is regulated by miR-193a-3p. FEBS J 286: 1136-1153, 2019.

29. Takahashi H, Takahashi M, Ohnuma S, Unno M, Yoshino Y, Ouchi K, Takahashi S, Yamada Y, Shimodaira H and Ishioka C: microRNA-193a-3p is specifically down-regulated and acts as a tumor suppressor in BRAF-mutated colorectal cancer. BMC Cancer 17: 723, 2017.

30. Mamoori A, Wahab R, Islam F, Lee K, Vider J, Lu CT, Gopalan V and Lam AK: Clinical and biological significance of miR-193a-3p targeted KRAS in colorectal cancer pathogenesis. Hum Pathol 71: 145-156, 2018.

31. Santarpia L, Calin GA, Adam L, Ye L, Fusco A, Giunti S, Thaller C, Paladini L, Zhang X, Jimenez C, et al: A miRNA signature associated with human metastatic medullary thyroid carcinoma. Endocr Relat Cancer 20: 809-823, 2013.

32. Baldin V, Lukas J, Marcote MJ, Pagano M and Draetta G: Cyclin D1 is a nuclear protein required for cell cycle progression in G1. Genes Dev 7: 812-821, 1993.

33. Zhao M, Xu P, Liu Z, Zhen Y, Chen Y, Liu Y, Fu Q, Deng X, Liang Z, Li Y, et al: Dual roles of miR-374a by modulated c-Jun respectively targets CCND1-inducing PI3K/AKT signal and PTEN-suppressing Wnt/ $\beta$-catenin signaling in non-small-cell lung cancer. Cell Death Dis 9: 78, 2018.

34. Li Y, Li D, Yang W, Fu H, Liu Y and Li Y: Overexpression of the transcription factor FOXP3 in lung adenocarcinoma sustains malignant character by promoting G1/S transition gene CCND1. Tumour Biol 37: 7395-7404, 2016.

35. Li Z, Wang C, Prendergast GC and Pestell RG: Cyclin D1 functions in cell migration. Cell Cycle 5: 2440-2442, 2006.

36. Huang H, Han Y, Yang X, Li M, Zhu R, Hu J, Zhang X, Wei R, Li K and Gao R: HNRNPK inhibits gastric cancer cell proliferation through $\mathrm{p} 53 / \mathrm{p} 21 / \mathrm{CCND} 1$ pathway. Oncotarget 8: 103364-103374, 2017. 
37. Xue J, Qin Z, Li X, Zhang J, Zheng Y, Xu W, Cao Q and Wang Z Genetic polymorphisms in cyclin D1 are associated with risk of renal cell cancer in the Chinese population. Oncotarget 8 : 80889-80899, 2017.

38. Cheng S, Serra S, Mercado M, Ezzat S and Asa SL: A high-throughput proteomic approach provides distinct signatures for thyroid cancer behavior. Clin Cancer Res 17: 2385-2394, 2011

39. Deng M, Brägelmann J, Schultze JL and Perner S: Web-TCGA: An online platform for integrated analysis of molecular cancer data sets. BMC Bioinformatics 17: 72, 2016.

40. Chandran UR, Medvedeva OP, Barmada MM, Blood PD Chakka A, Luthra S, Ferreira A, Wong KF, Lee AV, Zhang Z, et al: TCGA Expedition: A Data Acquisition and Management System for TCGA Data. PLoS One 11: e0165395, 2016.

41. Barrett T, Wilhite SE, Ledoux P, Evangelista C, Kim IF Tomashevsky M, Marshall KA, Phillippy KH, Sherman PM, Holko M, et al: NCBI GEO: Archive for functional genomics data sets - update. Nucleic Acids Res 41D: D991-D995, 2013.

42. Lassalle S, Zangari J, Popa A, Ilie M, Hofman V, Long E, Patey M, Tissier F, Belléannée $\mathrm{G}$, Trouette $\mathrm{H}$, et al: MicroRNA-375/SEC23A as biomarkers of the in vitro efficacy of vandetanib. Oncotarget 7: 30461-30478, 2016.

43. Minna E, Romeo P, Dugo M, De Cecco L, Todoerti K, Pilotti S, Perrone F, Seregni E, Agnelli L, Neri A, et al: miR-451a is underexpressed and targets AKT/mTOR pathway in papillary thyroid carcinoma. Oncotarget 7: 12731-12747, 2016.

44. Rossing M, Borup R, Henao R, Winther O, Vikesaa J, Niazi O Godballe C, Krogdahl A, Glud M, Hjort-Sørensen C, et al: Down-regulation of microRNAs controlling tumourigenic factors in follicular thyroid carcinoma. J Mol Endocrinol 48 : 11-23, 2012.

45. Ioannidis JP, Patsopoulos NA and Evangelou E: Uncertainty in heterogeneity estimates in meta-analyses. BMJ 335: 914-916, 2007.

46. Gan BL, He RQ, Zhang Y, Wei DM, Hu XH and Chen G: Downregulation of HOXA3 in lung adenocarcinoma and its relevant molecular mechanism analysed by RT-qPCR, TCGA and in silico analysis. Int J Oncol 53: 1557-1579, 2018

47. Deng Y, He R, Zhang R, Gan B, Zhang Y, Chen G and Hu X: The expression of HOXA13 in lung adenocarcinoma and its clinical significance: A study based on The Cancer Genome Atlas, Oncomine and reverse transcription-quantitative polymerase chain reaction. Oncol Lett 15: 8556-8572, 2018.

48. Liang YY, Huang JC, Tang RX, Chen WJ, Chen P, Cen WL, Shi K, Gao L, Gao X, Liu AG, et al: Clinical value of miR-198-5p in lung squamous cell carcinoma assessed using microarray and RT-qPCR. World J Surg Oncol 16: 22, 2018.

49. Ritchie ME, Phipson B, Wu D, Hu Y, Law CW, Shi W and Smyth GK: limma powers differential expression analyses for RNA-sequencing and microarray studies. Nucleic Acids Res 43: e47, 2015.

50. Bardou P, Mariette J, Escudié F, Djemiel C and Klopp C: jvenn: An interactive Venn diagram viewer. BMC Bioinformatics 15 : 293, 2014

51. The Gene Ontology Consortium: The Gene Ontology Resource: 20 years and still GOing strong. Nucleic Acids Res 47 (D1) D330-D338, 2019.

52. Kanehisa M, Sato Y, Furumichi M, Morishima K and Tanabe M: New approach for understanding genome variations in KEGG Nucleic Acids Res 47D: D590-D595, 2019.

53. Schriml LM, Mitraka E, Munro J, Tauber B, Schor M, Nickle L, Felix V, Jeng L, Bearer C, Lichenstein R, et al: Human Disease Ontology 2018 update: Classification, content and workflow expansion. Nucleic Acids Res 47D: D955-D962, 2019.

54. Yu G, Wang LG, Han Y and He QY: clusterProfiler: An R package for comparing biological themes among gene clusters. OMICS 16: 284-287, 2012

55. Wickham H (ed.): ggplot2: Elegant Graphics for Data Analysis Springer, New York, NY, 2016

56. Szklarczyk D, Gable AL, Lyon D, Junge A, Wyder S, Huerta-Cepas J, Simonovic M, Doncheva NT, Morris JH, Bork P, et al: STRING v11: Protein-protein association networks with increased coverage, supporting functional discovery in genome-wide experimental datasets. Nucleic Acids Res 47D: D607-D613, 2019.

57. Vasko V,Espinosa AV, Scouten W, He H, Auer H,Liyanarachchi S, Larin A, Savchenko V, Francis GL, de la Chapelle A, et al: Gene expression and functional evidence of epithelial-to-mesenchymal transition in papillary thyroid carcinoma invasion. Proc Natl Acad Sci USA 104: 2803-2808, 2007.
58. Fontaine JF, Mirebeau-Prunier D, Franc B, Triau S, Rodien P, Houlgatte R, Malthièry Y and Savagner F: Microarray analysis refines classification of non-medullary thyroid tumours of uncertain malignancy. Oncogene 27: 2228-2236, 2008.

59. Salvatore G, Nappi TC, Salerno P, Jiang Y, Garbi C, Ugolini C, Miccoli P, Basolo F, Castellone MD, Cirafici AM, et al: A cell proliferation and chromosomal instability signature in anaplastic thyroid carcinoma. Cancer Res 67: 10148-10158, 2007.

60. Giordano TJ, Au AY, Kuick R, Thomas DG, Rhodes DR, Wilhelm KG Jr, Vinco M, Misek DE, Sanders D, Zhu Z, et al: Delineation, functional validation, and bioinformatic evaluation of gene expression in thyroid follicular carcinomas with the PAX8-PPARG translocation. Clin Cancer Res 12: 1983-1993, 2006.

61. DomG,TarabichiM,UngerK,ThomasG,Oczko-WojciechowskaM, Bogdanova T, Jarzab B, Dumont JE, Detours V and Maenhaut C: A gene expression signature distinguishes normal tissues of sporadic and radiation-induced papillary thyroid carcinomas. Br J Cancer 107: 994-1000, 2012.

62. Handkiewicz-Junak D, Swierniak M, Rusinek D, Oczko-Wojciechowska M, Dom G, Maenhaut C, Unger K, Detours V, Bogdanova T, Thomas G, et al: Gene signature of the post-Chernobyl papillary thyroid cancer. Eur J Nucl Med Mol Imaging 43: 1267-1277, 2016.

63. Barros-Filho MC, Marchi FA, Pinto CA, Rogatto SR and Kowalski LP: High Diagnostic Accuracy Based on CLDN10, HMGA2, and LAMB3 Transcripts in Papillary Thyroid Carcinoma. J Clin Endocrinol Metab 100: E890-E899, 2015.

64. Pita JM, Figueiredo IF, Moura MM, Leite V and Cavaco BM: Cell cycle deregulation and TP53 and RAS mutations are major events in poorly differentiated and undifferentiated thyroid carcinomas. J Clin Endocrinol Metab 99: E497-E507, 2014.

65. Pita JM, Banito A, Cavaco BM and Leite V: Gene expression profiling associated with the progression to poorly differentiated thyroid carcinomas. Br J Cancer 101: 1782-1791, 2009.

66. Rusinek D, Swierniak M, Chmielik E, Kowal M, Kowalska M, Cyplinska R, Czarniecka A, Piglowski W, Korfanty J, Chekan M, et al: BRAFV600E-Associated Gene Expression Profile: Early Changes in the Transcriptome, Based on a Transgenic Mouse Model of Papillary Thyroid Carcinoma. PLoS One 10: e0143688, 2015.

67. von Roemeling CA, Marlow LA, Pinkerton AB, Crist A, Miller J, Tun HW, Smallridge RC and Copland JA: Aberrant lipid metabolism in anaplastic thyroid carcinoma reveals stearoyl CoA desaturase 1 as a novel therapeutic target. J Clin Endocrinol Metab 100: E697-E709, 2015.

68. Livak KJ and Schmittgen TD: Analysis of relative gene expression data using real-time quantitative PCR and the 2(-Delta Delta C(T)) Method. Methods 25: 402-408, 2001.

69. Liu J, Xu J, Li H, Sun C, Yu L, Li Y, Shi C, Zhou X, Bian X, Ping Y, et al: miR-146b-5p functions as a tumor suppressor by targeting TRAF6 and predicts the prognosis of human gliomas. Oncotarget 6: 29129-29142,2015.

70. Xiong DD, Li ZY, Liang L, He RQ, Ma FC, Luo DZ, Hu XH and Chen G: The LncRNA NEAT1 Accelerates Lung Adenocarcinoma Deterioration and Binds to Mir-193a-3p as a Competitive Endogenous RNA. Cell Physiol Biochem 48: 905-918, 2018.

71. Uhlén M, Fagerberg L, Hallström BM, Lindskog C, Oksvold P, Mardinoglu A, Sivertsson Å, Kampf C, Sjöstedt E, Asplund A, et al: Proteomics. Tissue-based map of the human proteome. Science 347: 1260419, 2015.

72. Kitahara CM and Sosa JA: The changing incidence of thyroid cancer. Nat Rev Endocrinol 12: 646-653, 2016.

73. Vigneri R, Malandrino P and Vigneri P: The changing epidemiology of thyroid cancer: Why is incidence increasing? Curr Opin Oncol 27: 1-7, 2015.

74. Raue F and Frank-Raue K: Thyroid Cancer: Risk-Stratified Management and Individualized Therapy. Clin Cancer Res 22: 5012-5021, 2016

75. Qiu J, Zhang W, Zang C, Liu X, Liu F, Ge R, Sun Y and Xia Q: Identification of key genes and miRNAs markers of papillary thyroid cancer. Biol Res 51: 45, 2018.

76. Nixon AM, Provatopoulou X, Kalogera E, Zografos GN and Gounaris A: Circulating thyroid cancer biomarkers: Current limitations and future prospects. Clin Endocrinol (Oxf) 87: 117-126, 2017.

77. Zhang Y, Pan J, Xu D, Yang Z, Sun J, Sun L, Wu Y and Qiao H: Combination of serum microRNAs and ultrasound profile as predictive biomarkers of diagnosis and prognosis for papillary thyroid microcarcinoma. Oncol Rep 40: 3611-3624, 2018. 
78. Nikiforov YE: Role Of Molecular Markers In Thyroid Nodule Management: Then And Now. Endocr Pract 23: 979-988, 2017.

79. Grossi I, Salvi A, Abeni E, Marchina E and De Petro G: Biological Function of MicroRNA193a-3p in Health and Disease. Int $\mathbf{J}$ Genomics 2017: 5913195, 2017.

80. Pekow J, Meckel K, Dougherty U, Huang Y, Chen X, Almoghrabi A, Mustafi R, Ayaloglu-Butun F, Deng Z, Haider HI, et al: miR-193a-3p is a Key Tumor Suppressor in Ulcerative Colitis-Associated Colon Cancer and Promotes Carcinogenesis through Upregulation of IL17RD. Clin Cancer Res 23: 5281-5291, 2017.

81. Fan Q, Hu X, Zhang H, Wang S, Zhang H, You C, Zhang CY, Liang H, Chen X and Ba Y: miR-193a-3p is an Important Tumour Suppressor in Lung Cancer and Directly Targets KRAS. Cell Physiol Biochem 44: 1311-1324, 2017.

82. Ren F, Ding H, Huang S, Wang H, Wu M, Luo D, Dang Y, Yang L and Chen G: Expression and clinicopathological significance of miR-193a-3p and its potential target astrocyte elevated gene-1 in non-small lung cancer tissues. Cancer Cell Int 15: 80, 2015.

83. Williams M, Kirschner MB, Cheng YY, Hanh J, Weiss J, Mugridge N, Wright CM, Linton A, Kao SC, Edelman JJ, et al miR-193a-3p is a potential tumor suppressor in malignant pleural mesothelioma. Oncotarget 6: 23480-23495, 2015.

84. Lin M, Duan B, Hu J, Yu H, Sheng H, Gao H and Huang J: Decreased expression of miR-193a-3p is associated with poor prognosis in colorectal cancer. Oncol Lett 14: 1061-1067, 2017.
85. Liu Y, Xu X, Xu X, Li S, Liang Z, Hu Z, Wu J, Zhu Y, Jin X, Wang X, et al: MicroRNA-193a-3p inhibits cell proliferation in prostate cancer by targeting cyclin D1. Oncol Lett 14: 5121-5128, 2017.

86. Liang W and Sun F: Identification of key genes of papillary thyroid cancer using integrated bioinformatics analysis. J Endocrinol Invest 41: 1237-1245, 2018.

87. Jeon S, Kim Y, Jeong YM, Bae JS and Jung CK: CCND1 Splice Variant as A Novel Diagnostic and Predictive Biomarker for Thyroid Cancer. Cancers (Basel) 10: 437, 2018.

88. Lamba Saini M, Weynand B, Rahier J, Mourad M, Hamoir M and Marbaix E: Cyclin D1 in well differentiated thyroid tumour of uncertain malignant potential. Diagn Pathol 10: 32, 2015.

89. Tsai KW, Leung CM, Lo YH, Chen TW, Chan WC, Yu SY, Tu YT, Lam HC, Li SC, Ger LP, et al: Arm Selection Preference of MicroRNA-193a Varies in Breast Cancer. Sci Rep 6: 28176, 2016.

This work is licensed under a Creative Commons Attribution-NonCommercial-NoDerivatives 4.0 International (CC BY-NC-ND 4.0) License. 